\title{
Mise au point
}

\section{Revue systématique des facteurs de risque modifiables des cancers de la muqueuse buccale}

\author{
Elise Arrivé ${ }^{1,2,{ }^{*}}$, Karine Ligier ${ }^{3}$, Morgane Trouillet ${ }^{1}$, Aida Mounkaila Harouna ${ }^{1}$, \\ Médéric de la Reberdière ${ }^{1}$, Jean-Christophe Fricain ${ }^{1,4}$ \\ 1 Université Victor Segalen, Bordeaux, France \\ 2 INSERM U897, Bordeaux, France \\ 3 Registre général des cancers de Lille et de sa région, Loos, France \\ 4 INSERM U1026, Bordeaux, France
}

(Reçu le $1^{\mathrm{er}}$ janvier 2012, accepté le 23 janvier 2012)

Mots clés :

cancer de la cavité buccale /

facteurs de risque

Key words: mouth neoplasm / risk factor

\begin{abstract}
Résumé - Les cancers de la cavité buccale (CCB) et des lèvres occupaient, en 2008 , le $16^{\text {ème }}$ rang des cancers dans le monde. Les principaux facteurs de risque connus de ces cancers sont le tabac et l'alcool mais il existe d'autres facteurs pouvant être ciblés dans le cadre d'une prévention primaire. L'objectif de cette étude était d'identifier les facteurs de risque évitables et les facteurs protecteurs des cancers de la muqueuse buccale à travers une revue systématique de la littérature scientifique récente. Les mots clés indexés dans le MeSH « risk factors » et « mouth neoplasms » ont été appliqués dans la base de données PubMed. Les articles publiés entre le 01/01/2005 et le 01/01/2011 en anglais et en français ont été retenus s'ils traitaient de CCB et d'un ou de plusieurs facteurs de risque. Les articles s'intéressant exclusivement aux cancers des glandes salivaires ou aux facteurs de risque génétiques, de même que les articles rapportant des études transversales ou de qualité insuffisante, ont été exclus. Sur 325 références, 32 ont été retenues : 19 études cas-témoins, 8 cohortes prospectives et 5 méta-analyses; 2 études concernaient uniquement le cancer des lèvres. Les principaux facteurs de risque mis en évidence étaient la consommation d'alcool et de tabac et leur effet synergique. Des facteurs génétiques, comme le polymorphisme du gène codant pour l'alcool déshydrogénase ont été identifiés comme cofacteurs. Le HPV 16 impliqué dans la survenue du cancer du col de l'utérus pourrait avoir un rôle oncogène sur la muqueuse buccale. Des études ont identifié des facteurs locaux (traumatisme, mauvaise hygiéne...) mais elles ne sont pas apparues suffisamment fiables méthodologiquement pour pouvoir s'appuyer sur leurs conclusions. L'exposition aux ultra-violets a été mise en cause dans la survenue du cancer des lèvres, principalement chez les sujets transplantés. Une association entre CCB et exposition professionnelle importante aux pesticides et à certains solvants a également été retrouvée. Au contraire, une alimentation riche en fruits et légumes frais, la consommation de café et les visites régulières chez le chirurgien-dentiste étaient associées à une diminution du risque de CCB.

Les CCB sont accessibles à une prévention primaire, axée aujourd'hui en France sur la lutte contre le tabagisme et la consommation d'alcool, qui s'intègre dans une démarche de santé publique plus globale car ces facteurs de risque sont communs à d'autres cancers et à d'autres maladies chroniques fréquentes. Dans cette logique, le programme de vaccination contre le HPV impliqué dans le cancer du col de l'utérus et le Programme National Nutrition Santé auront peut-être un effet favorable sur l'incidence des CCB. En ce qui concerne la prévention secondaire, le dépistage des lésions précancéreuses ou de lésions invasives à un stade précoce fait l'objet de mesures dans le Plan cancer gouvernemental 2009-2013.
\end{abstract}

\begin{abstract}
Modifiable risk factors for mucous oral cancers: a systematic review. Cancers of oral cavity and lips were, in 2008, the 16 th most common cancer worldwide. The main known risk factors for these cancers are tobacco and alcohol consumption but there are other factors that may be targeted by primary prevention. The objective of this study was to identify preventable risk factors and protective factors for cancers of the oral mucosa through a systematic review of the recent scientific literature. The key words from the MeSH database "risk factors" and "mouth neoplasms" were applied to the PubMed database. Articles published between 01/01/2005 and 01/01/2011 in English and French were selected if they dealt with cancer of the oral cavity and one or more expositions. Articles
\end{abstract}

* Correspondance : elise.arrive@u-bordeaux2.fr 
focused exclusively on cancer of the salivary glands or genetic factors, as well as articles relating cross-sectional studies or of insufficient quality, were excluded. Of 325 references, 32 were included: 19 case-control studies, 8 prospective cohorts and 5 meta-analyses; 2 studies concerned only cancer of the lips. The main risk factors identified were alcohol and tobacco consumption and their synergistic effect. Genetic factors, such as polymorphism of the gene encoding for alcohol dehydrogenase have been identified as cofactors. The HPV 16 subtype involved in the cervical cancer could have an oncogene role on the oral mucosa. Studies have identified local factors (trauma, poor hygiene...) but they did not appear reliable enough methodically to support strong conclusions. Exposure to ultraviolet radiation has been implicated in the occurrence of lip cancer, especially among transplant recipients. An association between oral cancer and important occupational exposure to pesticides and to some solvents has also been found. On the contrary, a diet rich in fruits and vegetables, coffee consumption and regular dental visits were associated with a decreased risk of oral cancer.

Cancers of the oral cavity are accessible by primary prevention which is focused today in France on the fight against smoking and alcohol consumption, which is part of a public health approach more comprehensive because these risk factors are common to other cancers or other common chronic diseases. In this logic, the immunization program against HPV implicated in cervical cancer and the National Health and Nutrition Program against obesity and nutritional disorders may have a favorable effect on the incidence of cancers of the oral cavity, but this remains to be evaluated.

Les cancers représentent la plus grande cause de morbidité et de mortalité dans le monde avec plus de 10 millions de cas incidents chaque année [1]. Les cancers de la cavité buccale (CCB) et des lèvres occupaient, en 2008, le $16^{\text {ème }}$ rang des cancers dans le monde avec environ 260000 cas incidents, soit un taux d'incidence standardisé (Monde) de 3,8 pour 100000 personnes-années (5,3 pour les hommes et 2,6 pour les femmes) [2]. Ces cancers étaient responsables d'environ 125000 décès. Ils sont plus fréquents dans les pays ayant des ressources limitées et touchent plutôt les hommes [3].

En 2005, en France métropolitaine, les cancers de la lèvre et les CCB étaient classés au $5^{\text {ème }}$ rang chez les hommes et au $15^{\text {ème }}$ rang chez les femmes, les taux d'incidence standardisés respectifs (Monde) étant de 12,3 et 3,0 pour 100000 personnes-années. Les projections d'incidence pour l'année 2010 montraient qu'environ 6500 nouveaux cas de cancers de la lèvre et de CCB seraient diagnostiqués [4].

En France, depuis les années 80, les taux d'incidence des CCB ont diminué d'environ $40 \%$ chez les hommes et augmenté de $50 \%$ chez les femmes [4]. Cette évolution est à mettre en relation avec les modifications intervenues dans l'exposition aux principaux facteurs de risque : le tabac et l'alcool.

Hormis les cancers des lèvres, les CCB sont de mauvais pronostic : la survie relative à 5 ans varie de 33 à $38 \%$ chez les hommes et de 45 à $53 \%$ chez les femmes selon les localisations [5]. Cette survie ne s'est pas amélioré pour les cas diagnostiqués ces dernières années [5].

Les principaux facteurs de risque connus pour les CCB sont le tabac et l'alcool. D'autres expositions ont été étudiées dont certaines pouvant être ciblées dans le cadre de mesures de prévention primaire. L'objectif de cette étude était d'identifier les facteurs de risque évitables et les facteurs protecteurs des CCB à travers une revue systématique de la littérature scientifique récente.

\section{Méthodes}

\section{Schéma de l'étude}

Une revue systématique de la littérature a été réalisée à partir de la base de données PubMed.

\section{Définition des mots clés}

Aucun facteur de risque n'a été défini au préalable pour éviter d'exclure les articles traitant de facteurs peu connus a priori.

La définition des mots clés a également été la plus large possible en utilisant le MeSH. La leucoplasie chevelue, lésion associée au VIH, a été exclue, de même que les articles concernant le dépistage et la prise en charge des cancers.

\section{Stratégie de recherche}

L'équation de mots-clés suivante a été utilisée :

(« risk factors » [Mesh] and « mouth neoplasms » [Mesh] not « leukoplakia, hairy » [Mesh]) not (« mouth neoplasms/ drug therapy »[Mesh] or " mouth neoplasms/radiotherapy » [Mesh] or « mouth neoplasms/surgery » [Mesh] or « mouth neoplasms/therapy »[Mesh]).

De plus, on a sélectionné uniquement les publications :

- en langue anglaise et française ;

- concernant les êtres humains ;

- parues entre le 01/01/2005 et le 01/01/2011;

La recherche a été réalisée en février 2011.

\section{Sélection des articles}

Cette recherche a permis d'obtenir une liste initiale (liste 0 ). Une première sélection des articles (liste 1 ) a été 
réalisée sur la base des titres et résumés à l'aide d'une grille de sélection construite à cet effet ; les articles traitant des CCB et d'un ou plusieurs facteurs de risque ont été considérés comme pertinents. Les articles traitant exclusivement des cancers des glandes salivaires ont été exclus car ce sont des cancers rares, ayant des facteurs de risque différents de ceux des CCB. Les articles traitant des facteurs de risque génétiques associés à un facteur de risque modifiable ont été inclus. Des critères portant sur le schéma d'étude et sur la qualité ont ensuite été appliqués pour obtenir une liste finale (liste 2). Les études transversales ont été exclues car c'est un schéma d'étude moins bien adapté à la recherche des facteurs de risque du fait des biais de sélection et du rapport de temps entre exposition et maladie. Les critères de lecture de Salmi et al. ont été appliqués pour évaluer la qualité des études (formulation claire de l'objectif, méthodes décrites et adéquates, prise en compte des variables importantes, discussion des critères de causalité...) [6].

\section{Extraction des données et synthèse}

Les informations concernant les études retenues ont été extraites à l'aide d'une grille d'extraction de données réalisée à cet effet, puis elles ont été synthétisées dans un tableau.

\section{Résultats}

La recherche a identifié 325 articles (liste 0 ). La liste 1 et la liste 2 en comptaient respectivement 78 et 32, après exclusion des 34 études hors critères, des 4 études transversales et des 8 de qualité insuffisante.

Sur les articles sélectionnés, 4 provenaient d'Amérique du Nord (dont 3 des États-Unis) [7-10], 13 d'Europe (dont 6 d'Italie et aucune de France) [11-23], 9 d'Asie (dont 4 de l'Inde et 4 du Japon) [24-32], 1 d'0céanie [33], 3 d'Amérique du Sud [34-36], et 2 correspondaient à des méta-analyses effectuées sur plusieurs continents [37, 38] (Tab. II). Dix-neuf études étaient de type cas-témoins, 8 de type cohortes prospectives $[17,20,24-26,28,32,33]$ et cinq de type méta-analyses [22, 29, 30, 36, 37, 38]. Deux études s'intéressaient uniquement au cancer des lèvres [12, 33], les autres aux CCB et/ou aux cancers des voies aéro-digestives supérieures.

Les principaux facteurs de risque étudiés étaient la consommation de tabac et la consommation d'alcool (Tab. I). Ils étaient systématiquement associés à un risque accru de CCB, et leur consommation simultanée avait un effet synergique avec des odds-ratios (OR) variant de 3,3 à 11,3 selon les études $[19,27,32]$. Une étude a même rapporté un OR de 34,8 pour une consommation élevée conjointe de tabac et d'alcool alors que les $O R$ estimés de manière séparée pour ces consommations s'élevaient à 6 environ [19]. Différentes formes de consommation de tabac ont été étudiées : tabac à chiquer, à priser, sous forme de bidîs (cigarettes indiennes contenant du tabac et/ ou des feuilles d'eucalyptus) (OR variant entre 1,9 et 4,6 ) ou associé à du bétel (plante médicinale retrouvée en Inde) avec un $\mathrm{OR}$ de $3,2(p<0,05)$ [27]. Toutes ces formes de consommation étaient plus fréquentes chez les sujets atteints d'un CCB. L'arrêt du tabac permettrait de diminuer le risque de CCB avec un $0 R$ décroissant de moitié par tranche de 10 ans après l'arrêt [21]. La consommation de marijuana n'était pas associée aux cas de CCB (OR ajusté = 1,1 [10]).

Plusieurs études montraient que la consommation d'alcool était un facteur de risque de CCB. Deux études ont évalué l'effet du polymorphisme du gène codant pour l'enzyme alcool déshydrogénase : certaines formes sont moins actives (ADH1C et $A D H 1 B$ ) et semblent constituer un facteur de risque chez les grands consommateurs d'alcool ( $O R$ variant de 4,3 à 9,3 pour la forme $2 \times 2$ de l'ADH1C et 4,2 pour la forme $1 \times 1$ de l'ADH1B) $[19,31]$. Un indice de masse corporel (IMC) bas $(<18,5)$ a été souvent retrouvé chez les sujets atteints d'un CCB $(0 R$ de 2,6) et augmenterait les $\mathrm{OR}$ liés à la consommation de tabac et d'alcool de manière significative ; les mécanismes biologiques responsables n'ont pas été identifiés [38]. La consommation de tabac et d'alcool étant un facteur de risque prédominant, elle constitue un facteur de confusion pour l'étude des autres expositions et elle apparaît quasiment systématiquement comme covariable dans les analyses statistiques multivariées (Tab. II).

La consommation de fruits et de légumes frais ( $O R$ variant de 0,34 à 0,67$)[13,14,35]$ ou de café (OR variant de 0,51 à $0,96)[24,37]$ était associée à un risque moins élevé de CCB contrairement à ce que l'on observe avec la consommation de viande et de légumes cuits (OR variant de 1,9 à 3,8$)[13,15$, $35]$, d'aliments frits $(O R$ de 1,1$)[13,15,35]$ ou de maté $(0 R$ de 2,1) [36]. Ce sont les flavonoïdes, métabolites naturellement présents dans les fruits et les légumes de l'alimentation, qui semblent jouer un rôle protecteur vis-à-vis des CCB grâce à leurs propriétés anti-oxydantes $(O R$ de 0,56$)$ [16]. Une étude a montré qu'un déficit en fer et une concentration sérique élevée en glutathion étaient respectivement un facteur de risque et un facteur protecteur (OR de 0,4) [7]. En effet, un déficit en fer semble induire un stress oxydatif générant des radicaux libres, entrainant des dommages cellulaires $(0 R$ de 8,25) [7], contrairement au glutathion qui est un anti-oxydant intra-cellulaire. Le café contiendrait également des composés chimiques ayant des effets anti-oxydants et anti-mutagènes [24, 37]. En particulier, la caféine pourrait inhiber l'activation de la croissance cellulaire ; le cafestol et le kahweol les dommages causés à l'ADN par certains procarcinogènes. Cependant, le mécanisme biologique sous-jacent n'est pas clairement établi $[24,37]$. Au contraire, le maté, une infusion traditionnelle sud-américaine de yerba maté, contiendrait des oncogènes potentiels, en particulier des hydrocarbures aromatiques polycycliques provenant de la fumée du bois utilisé pour son séchage [36]. 
Tableau I. Synopsis des principaux facteurs étudiés dans la survenue des cancers buccaux (2005-2010). Table I. Synopsis of principle factors studied in the developmentof oral cancers (2005-2010).

\begin{tabular}{|c|c|c|c|}
\hline \multirow[t]{2}{*}{ Facteur étudié } & \multicolumn{3}{|c|}{ Conclusion } \\
\hline & Risque & Protection & Non significatif \\
\hline $\begin{array}{l}\text { Consommation de tabac et autres } \\
\text { En général } \\
\text { A chiquer } \\
\text { A priser } \\
\text { Bidîs } \\
\text { Bétel } \\
\text { Marijuana }\end{array}$ & $\begin{array}{l}{[9,21-23,27,32]} \\
{[28,29]} \\
{[23]} \\
{[27,29,30]} \\
{[27]}\end{array}$ & Arrêt [21] & {$[10]$} \\
\hline $\begin{array}{l}\text { Consommation d'alcool et susceptibilité génétique } \\
\text { Consommation d'alcool } \\
\text { ADH1C } \\
\text { ADH1B }\end{array}$ & $\begin{array}{l}{[9,20,23,26,27,32]} \\
{[19,31]} \\
{[31]}\end{array}$ & & \\
\hline $\begin{array}{l}\text { Alimentation } \\
\text { Consommation de fruits et légumes frais } \\
\text { Consommation de viande et légumes cuits/frits } \\
\text { Consommation de produits laitiers } \\
\text { Consommation de café } \\
\text { Consommation de thé } \\
\text { Consommation de café décaféiné } \\
\text { Consommation de maté } \\
\text { IMC bas } \\
\text { GSH sérique élevé } \\
\text { Fer sérique bas } \\
\text { Flavonoïdes }\end{array}$ & $\begin{array}{l}{[36]} \\
{[38]} \\
{[7]}\end{array}$ & $\begin{array}{l}{[13,14,35]} \\
{[24,37]}\end{array}$ & $\begin{array}{l}{[13]} \\
{[25,37]} \\
{[37]}\end{array}$ \\
\hline $\begin{array}{l}\text { Condition et habitudes bucco-dentaires } \\
\text { Traumatisme chronique } \\
\text { Hygiène bucco-dentaire insuffisante } \\
\text { Nombre de dents absentes important } \\
\text { Visites régulières de contrôle bucco-dentaire }\end{array}$ & $\begin{array}{l}{[34]} \\
{[18]} \\
{[18]}\end{array}$ & [18] & \\
\hline $\begin{array}{l}\text { Divers } \\
\text { HPV } \\
\text { Travail à l'extérieur } \\
\text { Traitement immunosuppresseur } \\
\text { Exposition aux pesticides } \\
\text { Expositions aux solvants }\end{array}$ & $\begin{array}{l}{[8,9,18]} \\
{[12]} \\
{[33]} \\
{[17]} \\
{[17]}\end{array}$ & & \\
\hline
\end{tabular}

Deux études ont évalué des facteurs locaux et bucco-dentaires. Elles ont montré notamment qu'un traumatisme chronique de la muqueuse buccale $(0 R$ de 5,5$)$ et une hygiène buccodentaire insuffisante $(0 R$ de 5,3$)$ étaient associés aux $C C B$, car ils étaient susceptibles de favoriser l'apparition de lésions de la muqueuse buccale pouvant se transformer en $C C B$; un contrôle régulier permettrait de réduire ce risque $(0 \mathrm{R}$ de 0,4$)[18,34]$.

Certains papillomavirus humains (HPV) ont un potentiel oncogène et ils jouent un rôle important dans le cancer du col de l'utérus. Le rôle de l'infection à HPV dans les CCB était traité dans deux études, surtout pour les types à haut risque (OR de 1,7 pour le HPV 16 [9] et OR de 4,8 pour les HPV 16, 18, 31, $33,35,39,45,51,52,56,58,59,68,73,82$ [8]). Une autre étude qui a évalué le rôle des HPV n'a pas montré d'association mais les résultats n'étaient pas présentés sous forme d'OR et ils étaient difficilement interprétables [18].
Des facteurs environnementaux peuvent être associés aux $C C B$, notamment le travail à l'extérieur pour les cancers des lèvres, liés à l'exposition aux UV [12], mais également l'exposition aux pesticides et à certains solvants [17]. Les traitements immunosuppresseurs prescrits dans les greffes d'organe seraient associés à un risque plus important de cancer des lèvres par le renforcement de l'action des UV [33]. En effet, ces traitements sensibiliseraient l'ADN aux UV ou inhiberait la réparation des dommages causés à l'ADN par les UV.

\section{Discussion}

Cette revue de la littérature portant sur six années permet de revisiter un grand nombre de facteurs de risque évitables des CCB. Les principaux demeurent le tabac et l'alcool, mais c'est leur action synergique qui était fréquemment étudiée. 


\begin{tabular}{|c|c|c|c|c|c|c|}
\hline 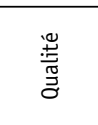 & 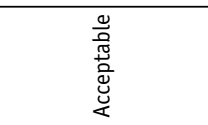 & 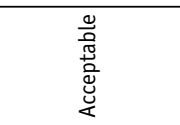 & $\begin{array}{l}\text { 营产 } \\
\text { 总 }\end{array}$ & $\begin{array}{l}\text { 蒡产 } \\
\text { 总 }\end{array}$ & 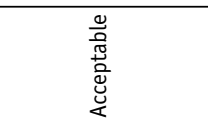 & 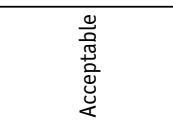 \\
\hline 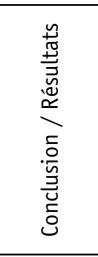 & 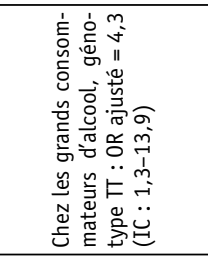 & 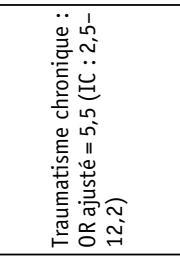 & 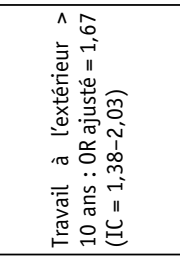 & 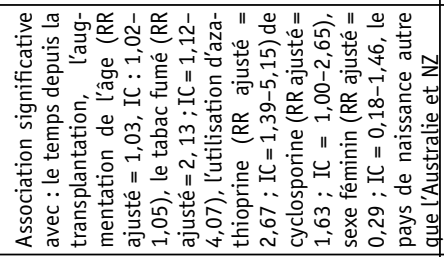 & 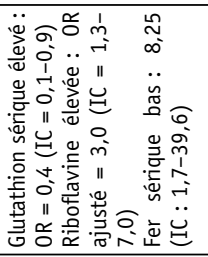 & 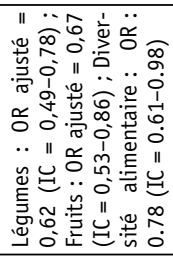 \\
\hline 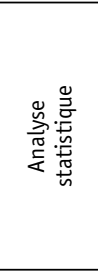 & 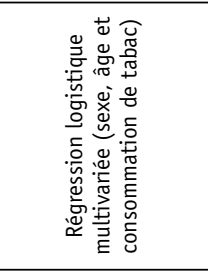 & 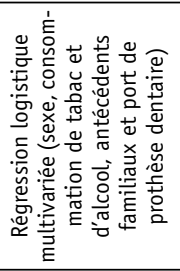 & 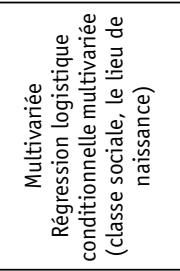 & 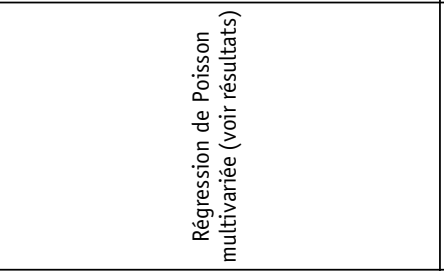 & 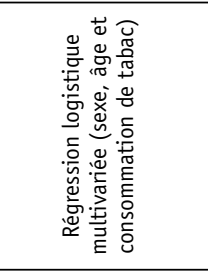 & 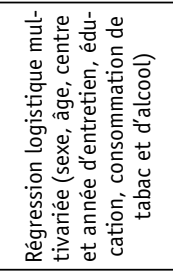 \\
\hline 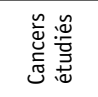 & 嵒 & 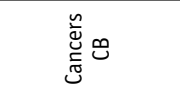 & 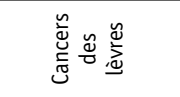 & 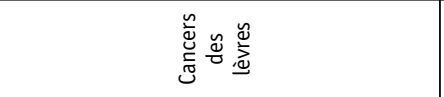 & 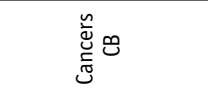 & 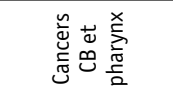 \\
\hline 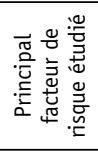 & 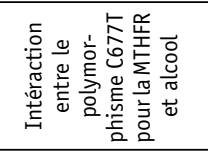 & 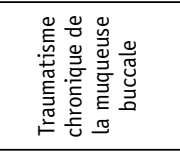 & 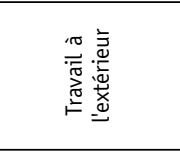 & 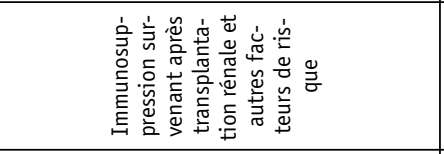 & 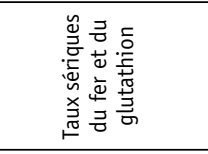 & 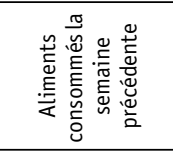 \\
\hline 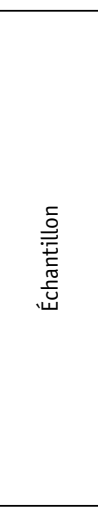 & 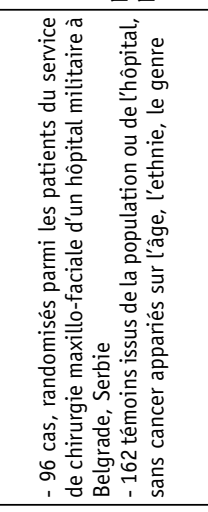 & 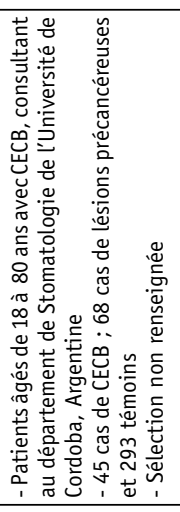 & 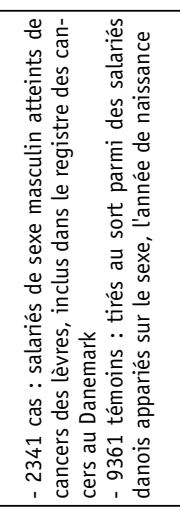 & 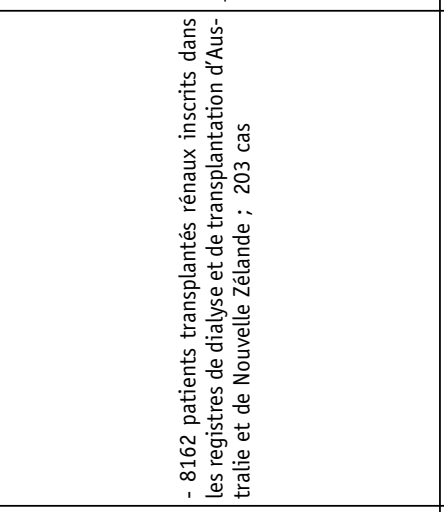 & 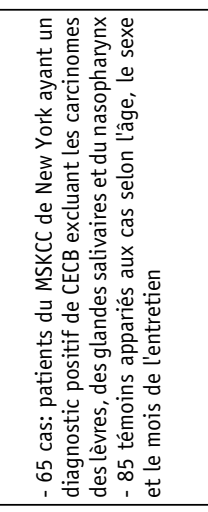 & 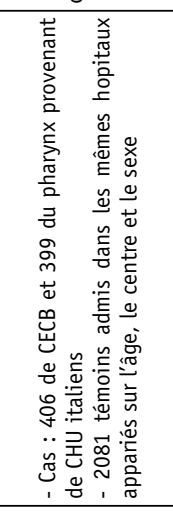 \\
\hline 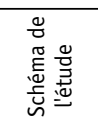 & 宸 & 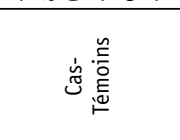 & 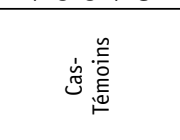 & 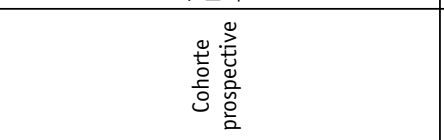 & 总言言 & 总言 \\
\hline $\begin{array}{l}\text { 壳 } \\
\text { 菏 }\end{array}$ & 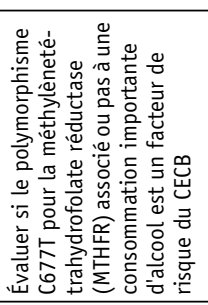 & 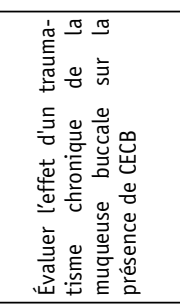 & 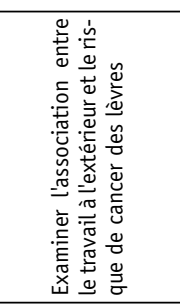 & 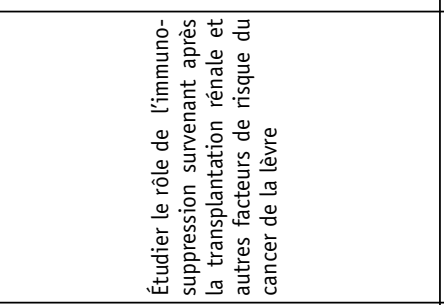 & 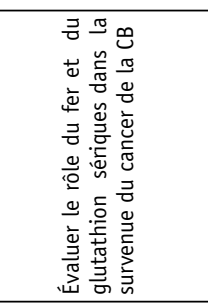 & 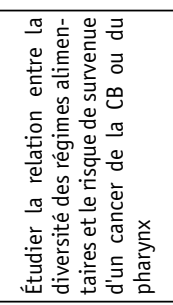 \\
\hline 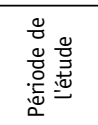 & 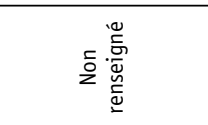 & $\begin{array}{l}\hat{\stackrel{\sim}{े}} \\
\text { ஸे } \\
\text { ò }\end{array}$ & 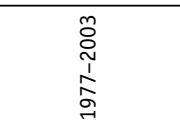 & 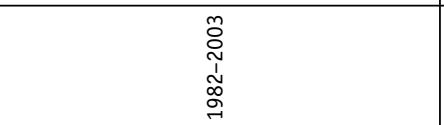 & 总 & 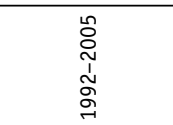 \\
\hline$\stackrel{g}{\stackrel{g}{E}}$ & 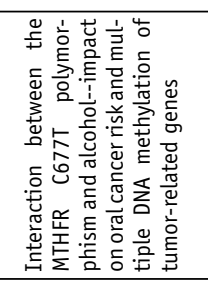 & 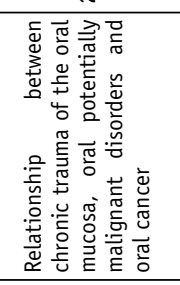 & 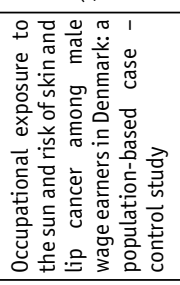 & 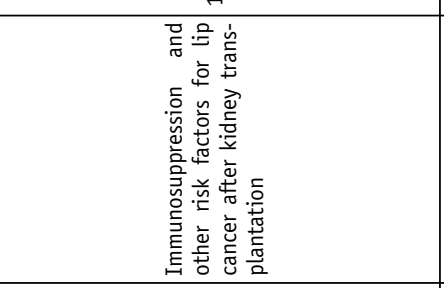 & 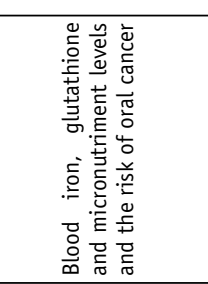 & 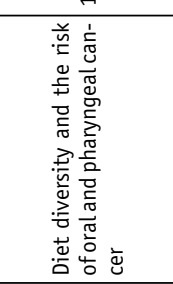 \\
\hline 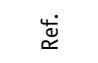 & $\Xi$ & F & 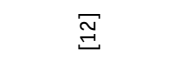 & 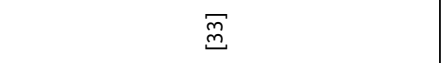 & $\Sigma$ & $\Xi$ \\
\hline
\end{tabular}




\begin{tabular}{|c|c|c|c|c|c|c|}
\hline 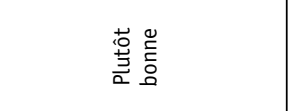 & 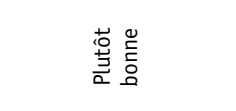 & \begin{tabular}{l} 
憙产 \\
\multirow{2}{*}{}
\end{tabular} & $\begin{array}{l}\text { 喜总 } \\
\text { 言 }\end{array}$ & $\begin{array}{l}\text { 咷产 } \\
\text { 总 }\end{array}$ & $\begin{array}{l}\text { 喜営 } \\
\text { 高 }\end{array}$ & 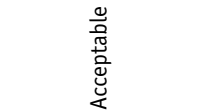 \\
\hline 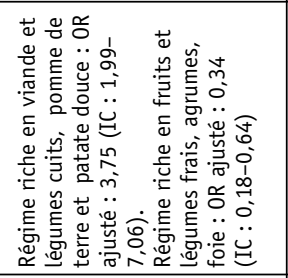 & 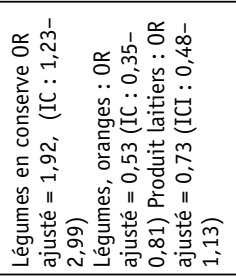 & 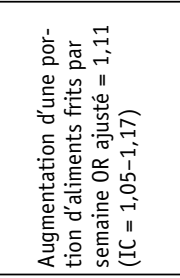 & 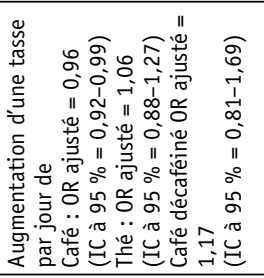 & 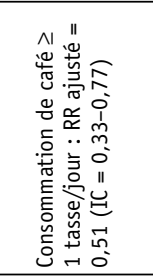 & 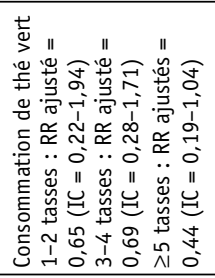 & 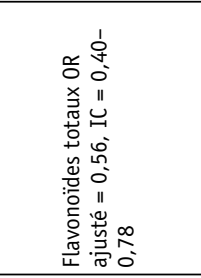 \\
\hline 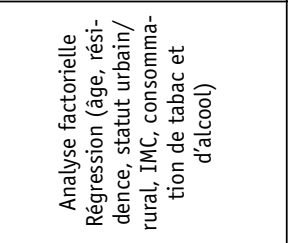 & 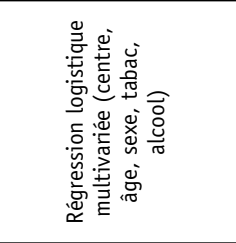 & 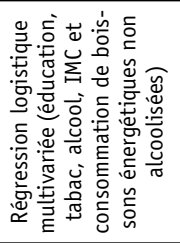 & 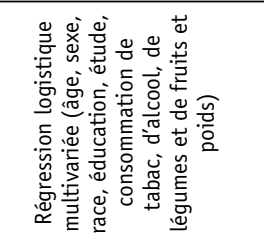 & 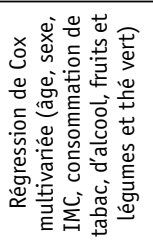 & 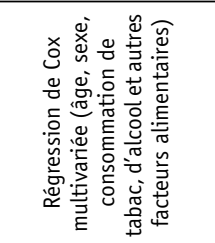 & 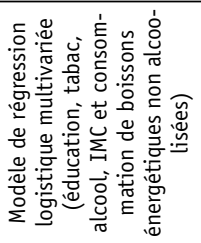 \\
\hline 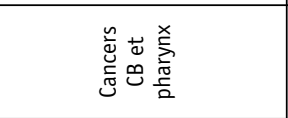 & 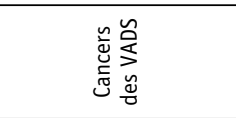 & 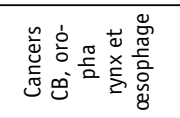 & 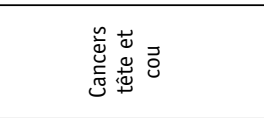 & 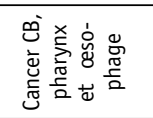 & 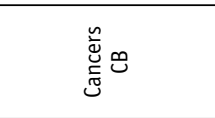 & 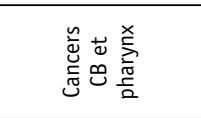 \\
\hline 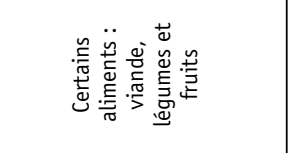 & 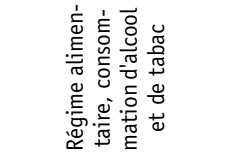 & 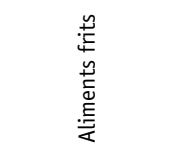 & 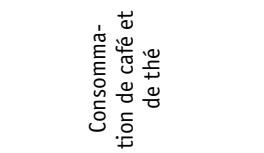 & 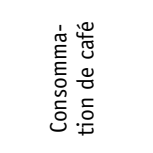 & 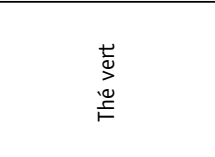 & 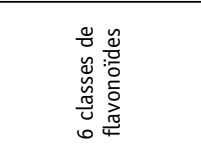 \\
\hline 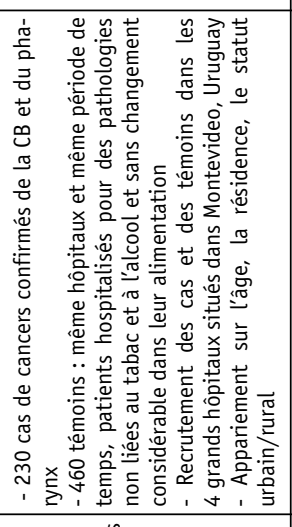 & 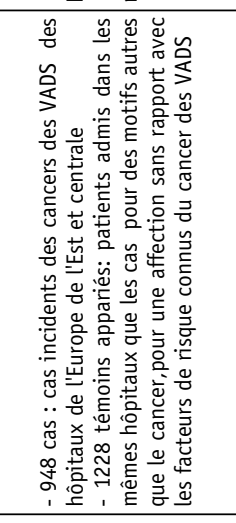 & 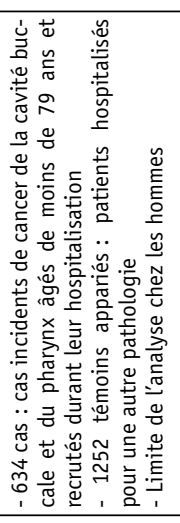 & 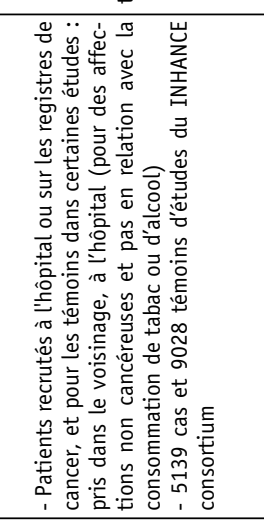 & 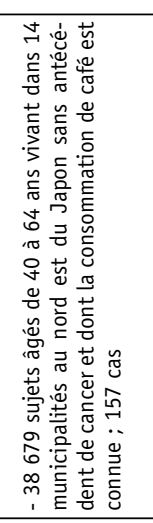 & 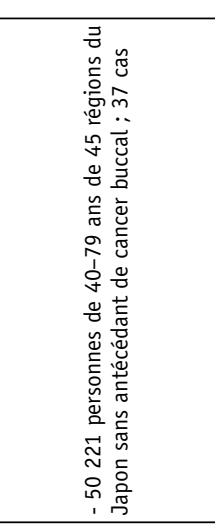 & 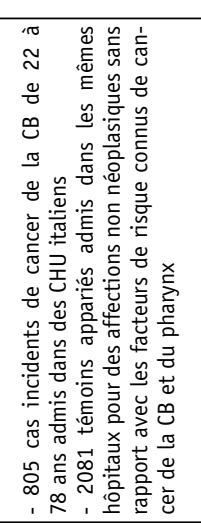 \\
\hline 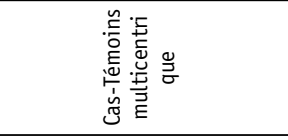 & 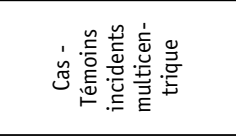 & 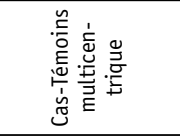 & 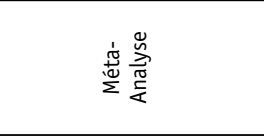 & 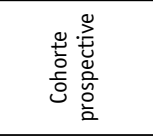 & 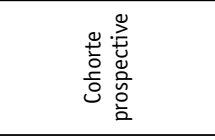 & 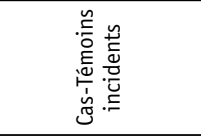 \\
\hline 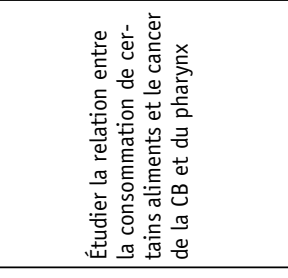 & 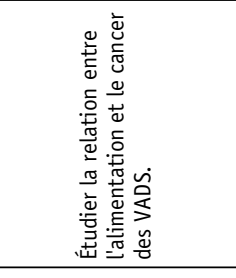 & 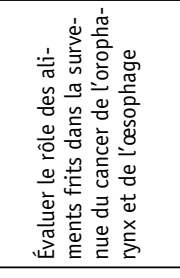 & 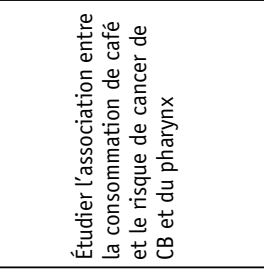 & 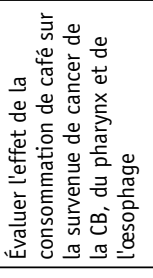 & 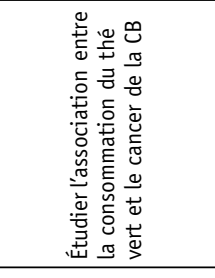 & 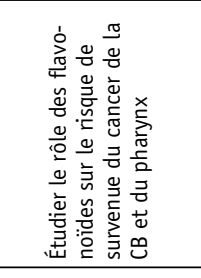 \\
\hline 客高 & 容愛 & 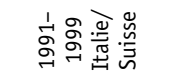 & 辛 & 客愛 & 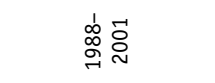 & 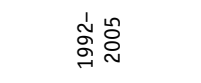 \\
\hline 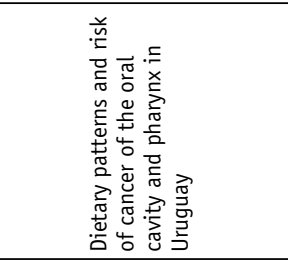 & 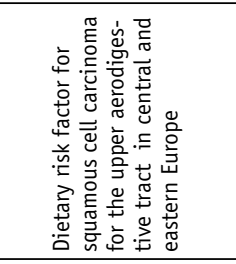 & 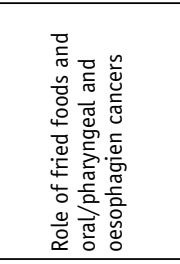 & 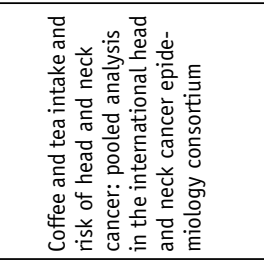 & 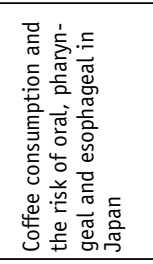 & 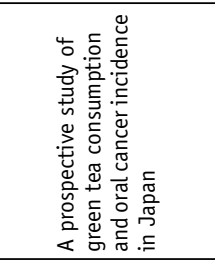 & 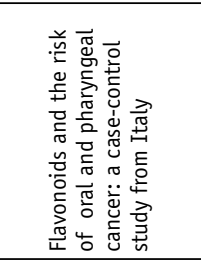 \\
\hline$\stackrel{\mathscr{m}}{\tilde{n}}$ & $\bar{\Xi}$ & 马્⿹ & $\stackrel{\Gamma}{\underline{m}}$ & $\underset{\Xi}{\mathbb{Z}}$ & 馬 & $\stackrel{\Xi}{\Xi}$ \\
\hline
\end{tabular}




\begin{tabular}{|c|c|c|c|c|c|}
\hline 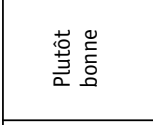 & 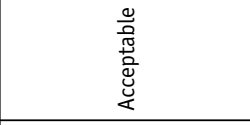 & 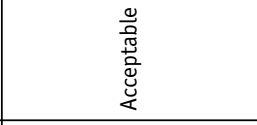 & 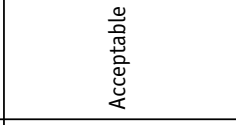 & 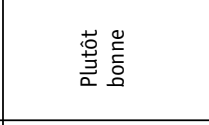 & 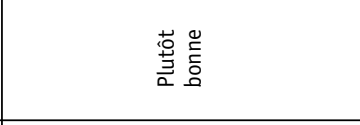 \\
\hline 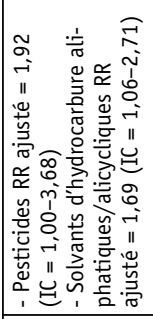 & 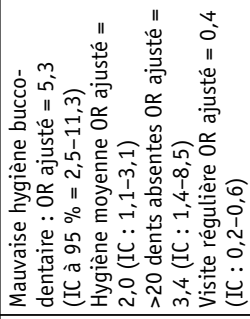 & 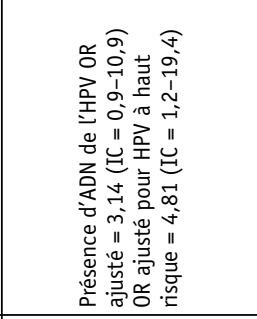 & 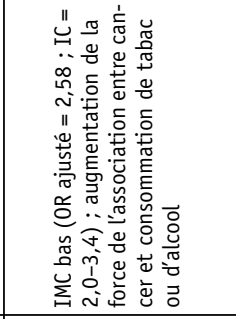 & 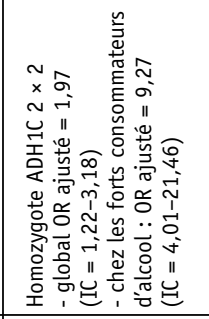 & 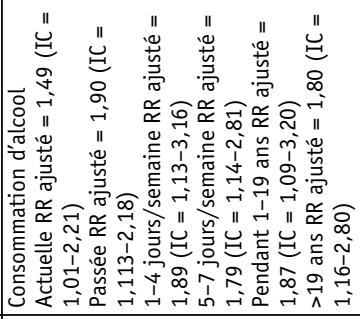 \\
\hline 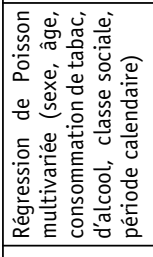 & 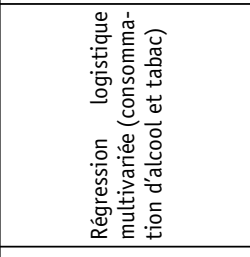 & 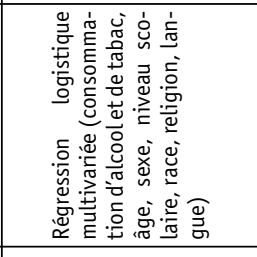 & 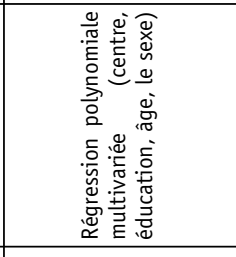 & 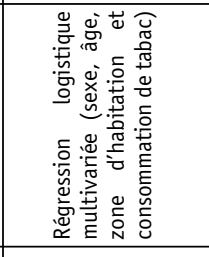 & 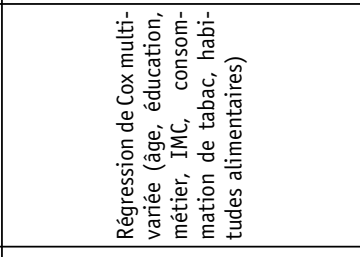 \\
\hline 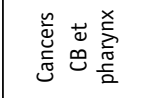 & 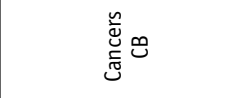 & 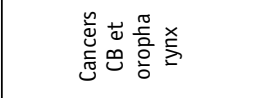 & & 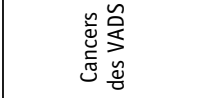 & 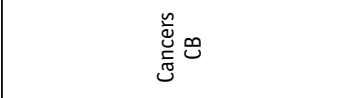 \\
\hline 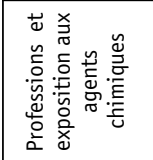 & 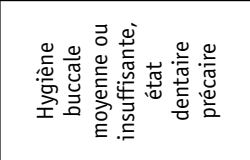 & 믐 & 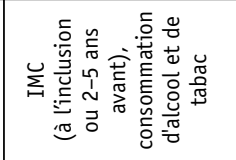 & 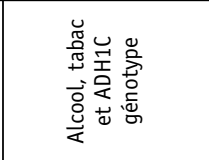 & 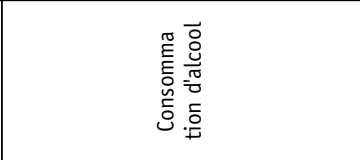 \\
\hline 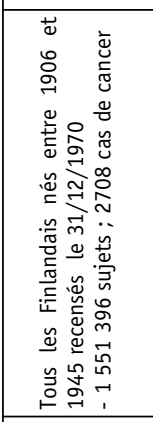 & 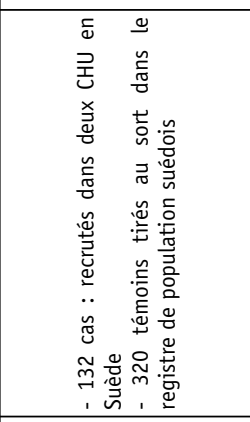 & 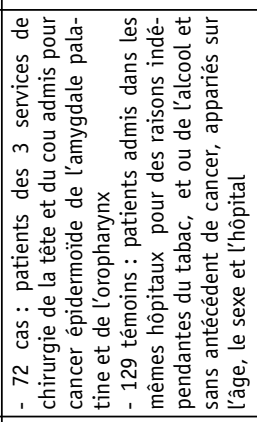 & 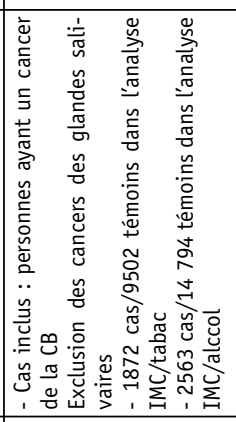 & 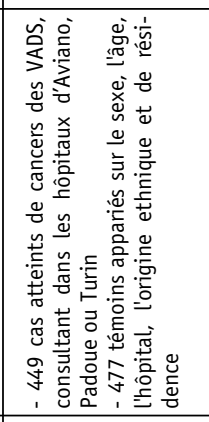 & 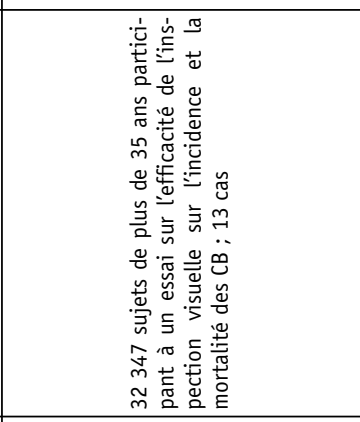 \\
\hline 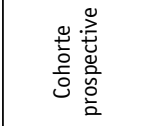 & 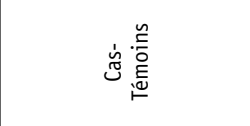 & 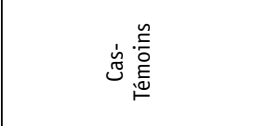 & 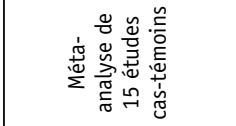 & 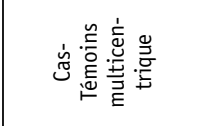 & 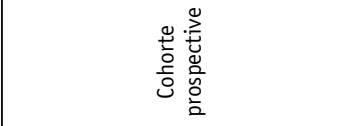 \\
\hline 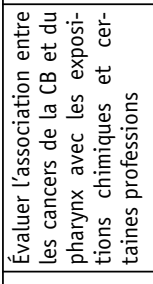 & 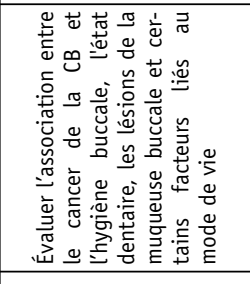 & 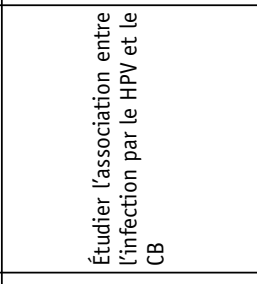 & 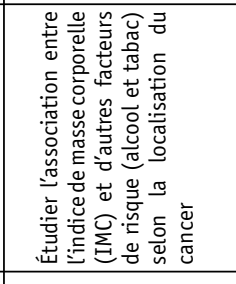 & 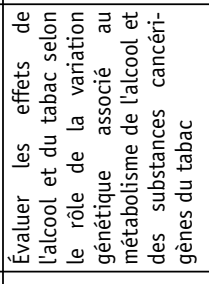 & 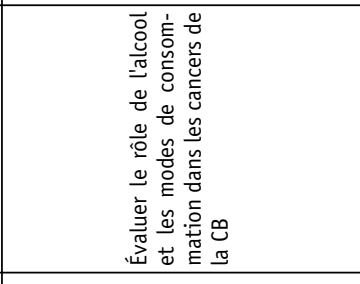 \\
\hline 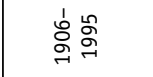 & - & 容客 & - & 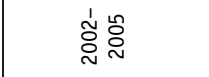 & $\begin{array}{l}1 \\
\text { 。ั } \\
\text { ò }\end{array}$ \\
\hline 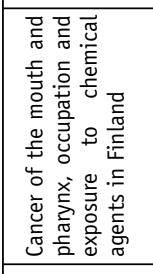 & 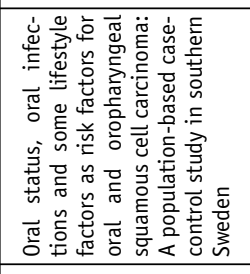 & 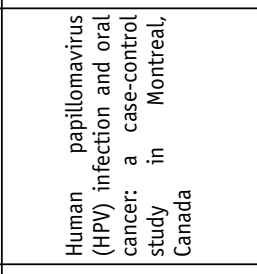 & 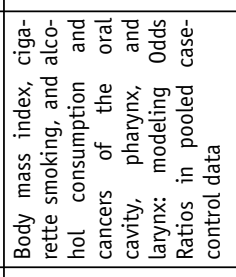 & 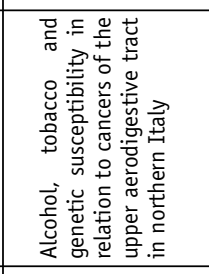 & 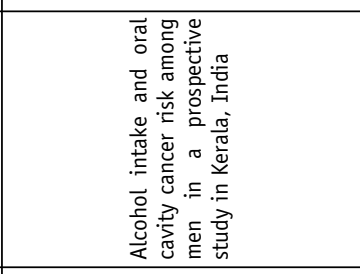 \\
\hline$\Xi$ & $\stackrel{\infty}{\Xi}$ & $\underline{\underline{\infty}}$ & 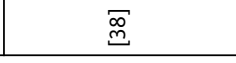 & פ & 胥 \\
\hline
\end{tabular}




\begin{tabular}{|c|c|c|c|c|c|}
\hline 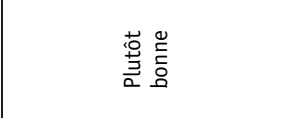 & 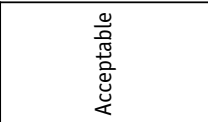 & $\begin{array}{l}\text { 营 总 } \\
\text { 咅 }\end{array}$ & 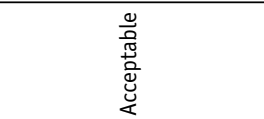 & 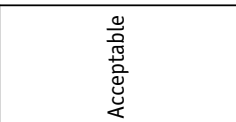 & $\begin{array}{l}\text { 珡高 } \\
\text { a }\end{array}$ \\
\hline 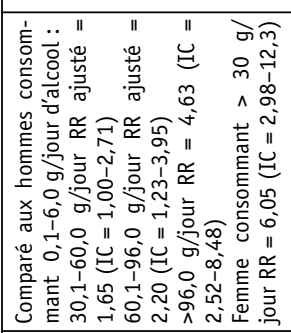 & 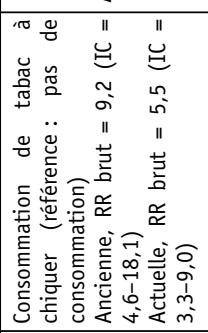 & 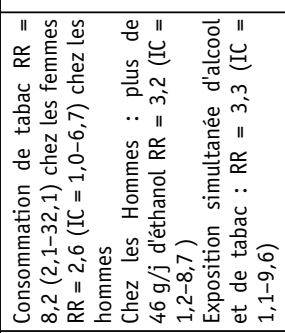 & 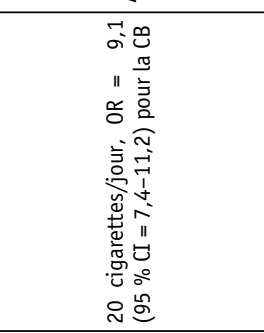 & 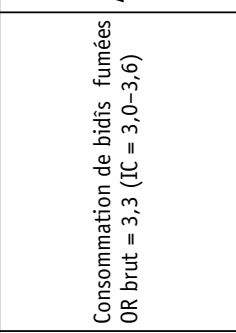 & 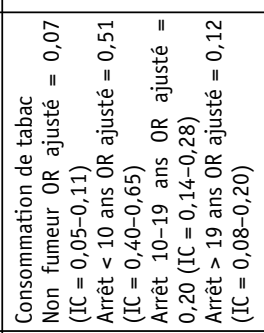 \\
\hline 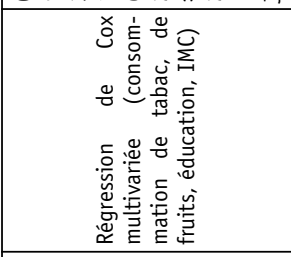 & 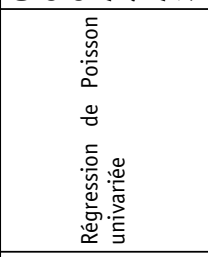 & 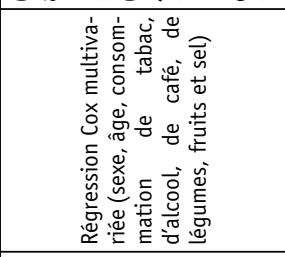 & 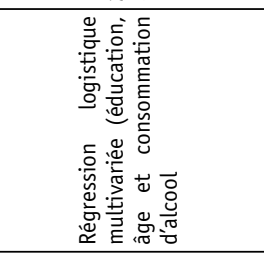 & 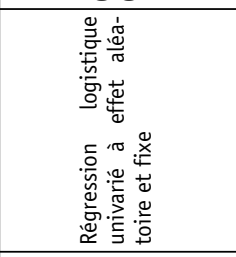 & 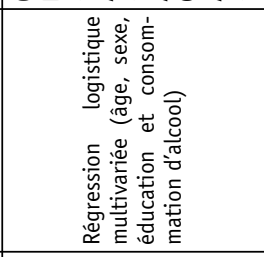 \\
\hline 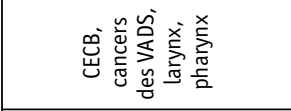 & 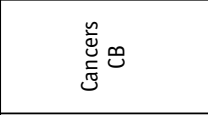 & 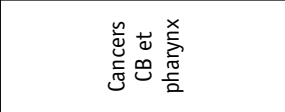 & 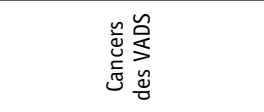 & 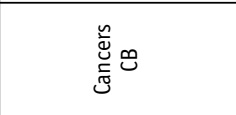 & 訔 \\
\hline 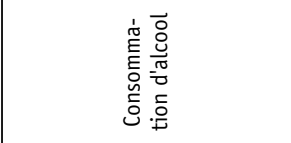 & 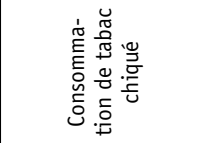 & 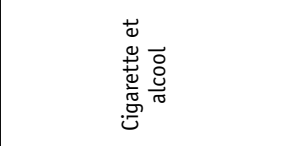 & 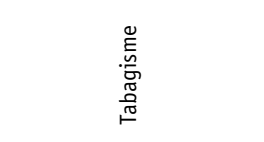 & 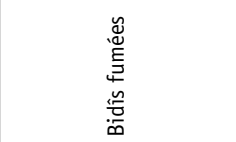 & 岕 \\
\hline 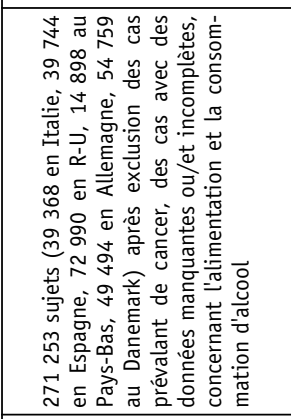 & 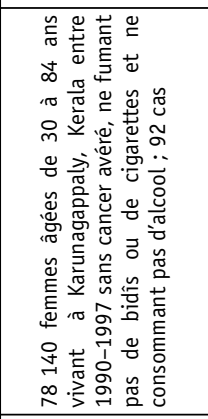 & 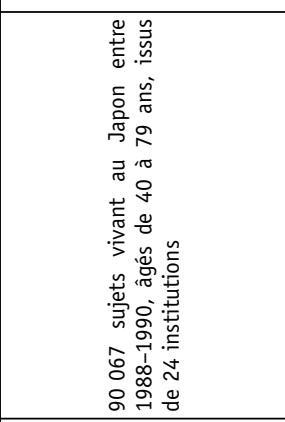 & 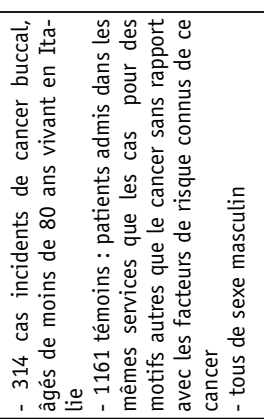 & 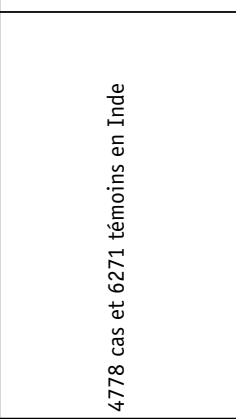 & 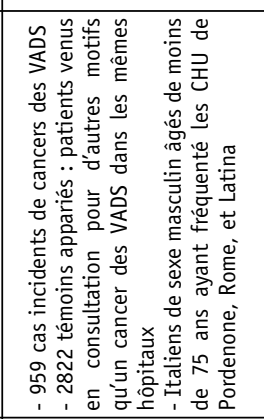 \\
\hline 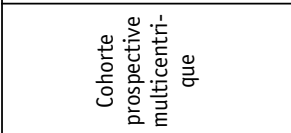 & 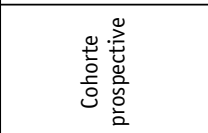 & 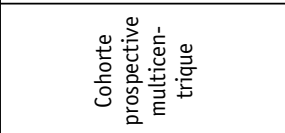 & 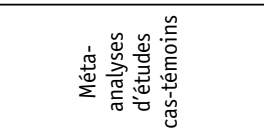 & 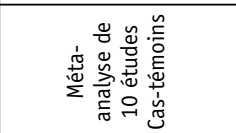 & 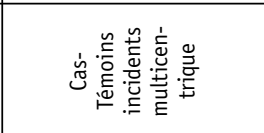 \\
\hline 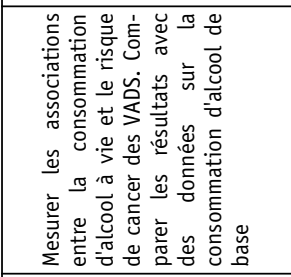 & 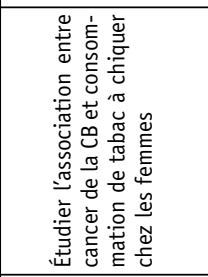 & 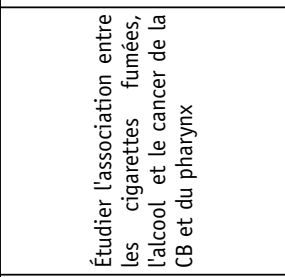 & 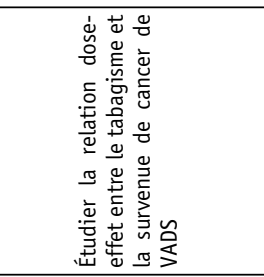 & 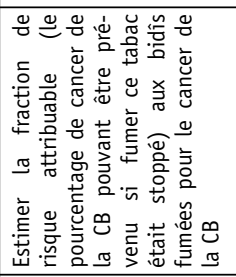 & 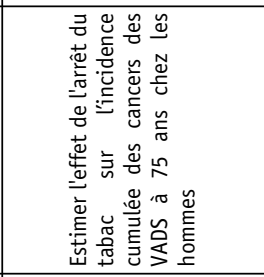 \\
\hline 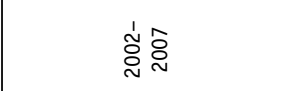 & 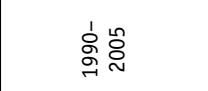 & 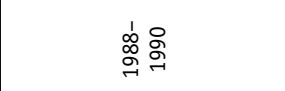 & 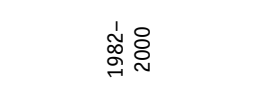 & 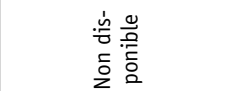 & $\begin{array}{l}1 \\
\vdots \\
\infty \\
0\end{array}$ \\
\hline 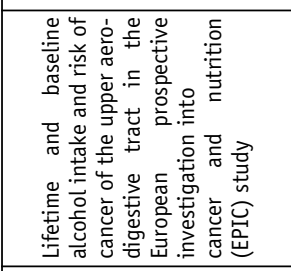 & 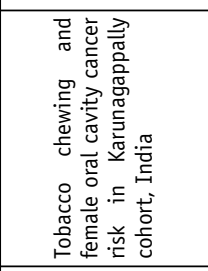 & 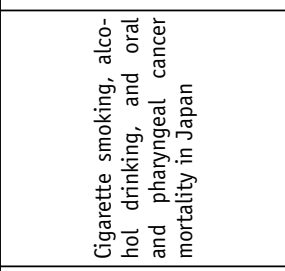 & 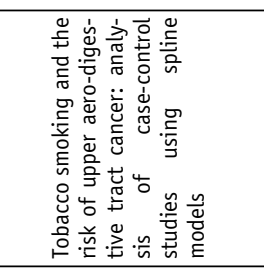 & 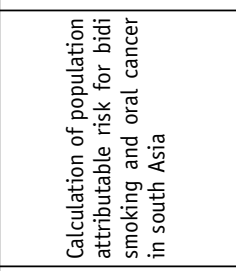 & 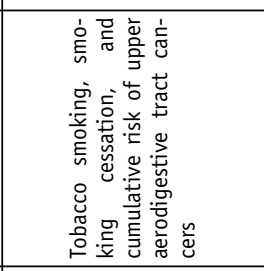 \\
\hline 离 & 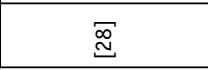 & $\widetilde{\widetilde{d}}$ & $\mathbb{\Xi}$ & $\bar{m}$ & $\vec{\Xi}$ \\
\hline
\end{tabular}




\begin{tabular}{|c|c|c|c|c|c|c|}
\hline 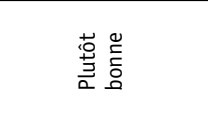 & $\begin{array}{l}\stackrel{0}{\bar{\Sigma}} \\
\stackrel{0}{\circ}\end{array}$ & 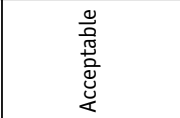 & 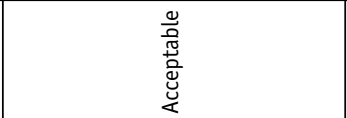 & 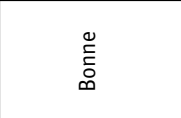 & $\begin{array}{l}\text { 总 } \\
\text { 总 }\end{array}$ & 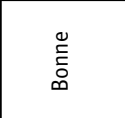 \\
\hline 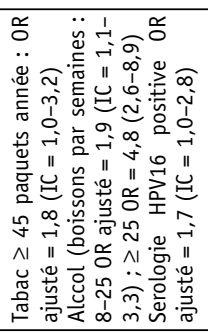 & 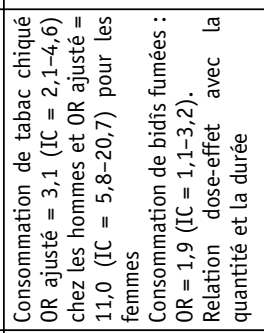 & 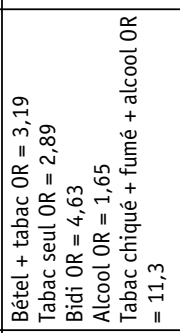 & 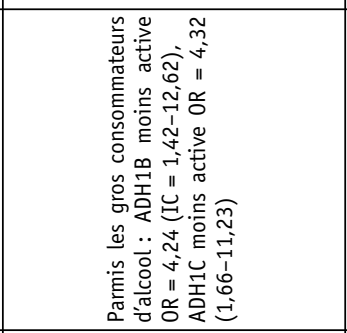 & 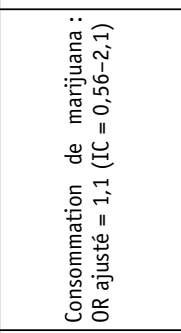 & 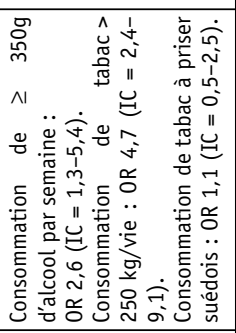 & 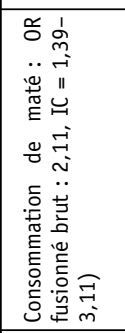 \\
\hline 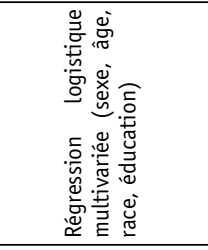 & 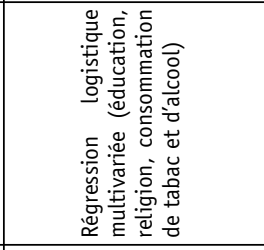 & 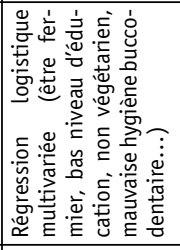 & 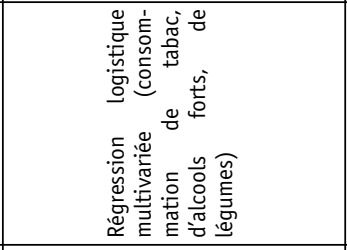 & 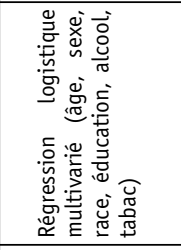 & 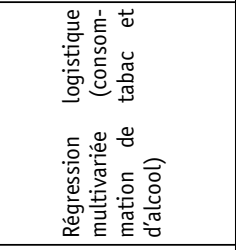 & 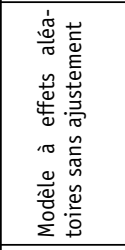 \\
\hline 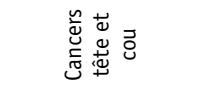 & 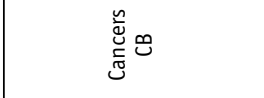 & 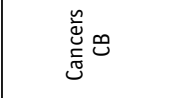 & 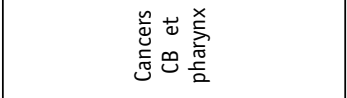 & 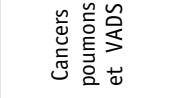 & 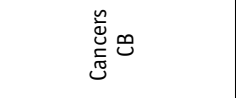 & 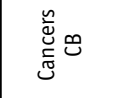 \\
\hline 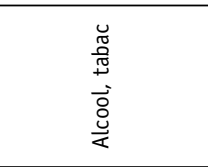 & 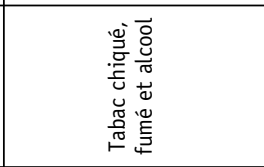 & 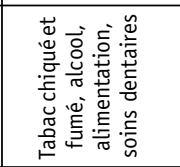 & 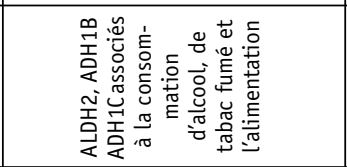 & 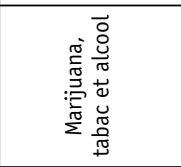 & 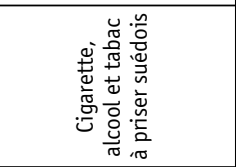 & 旁 \\
\hline 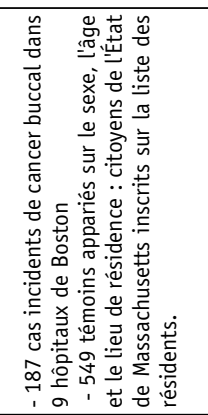 & 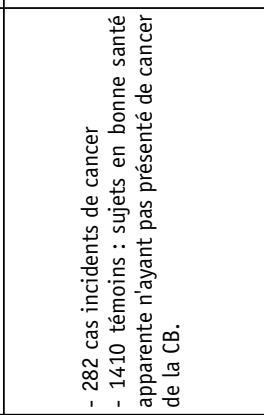 & 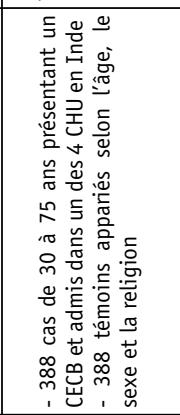 & 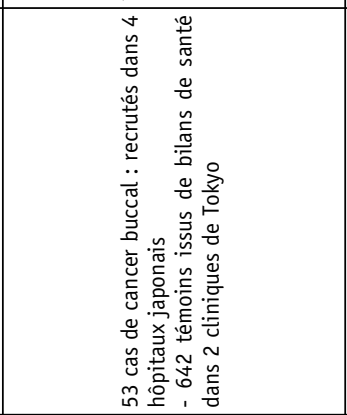 & 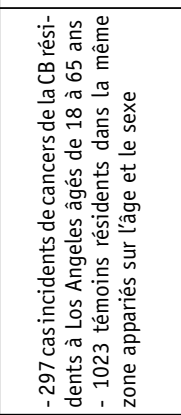 & 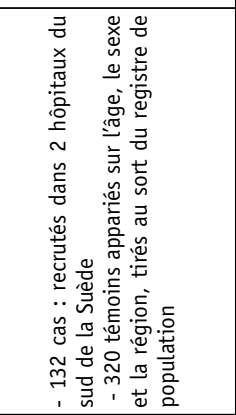 & 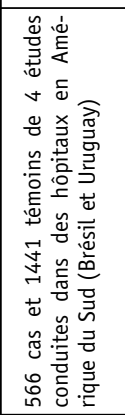 \\
\hline 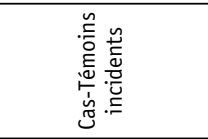 & 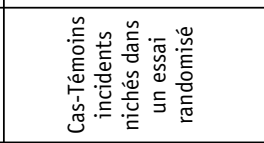 & 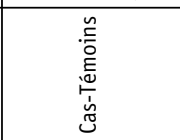 & 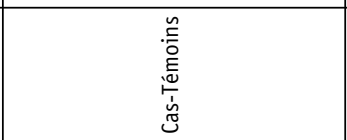 & 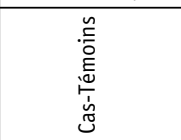 & 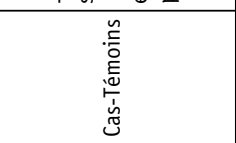 & 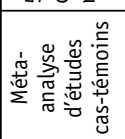 \\
\hline 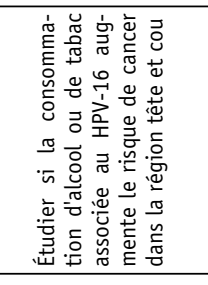 & 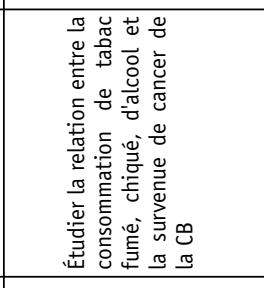 & 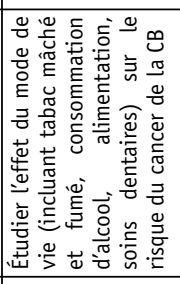 & 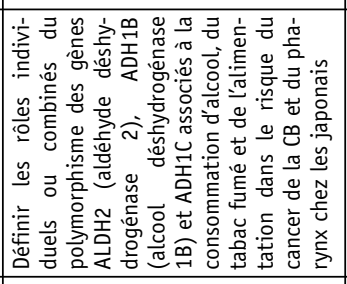 & 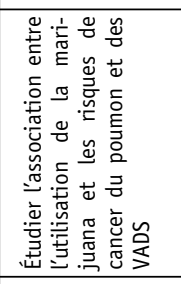 & 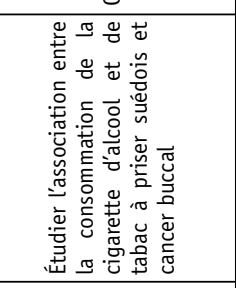 & 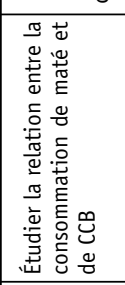 \\
\hline S̀ㅇㅇㅇ & 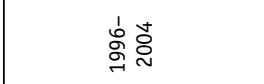 & 寄受 & 客 & 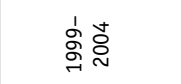 & 客总 & 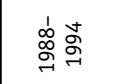 \\
\hline 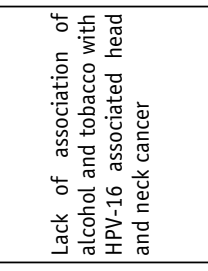 & 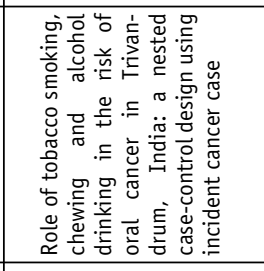 & 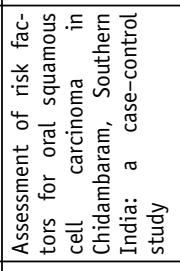 & 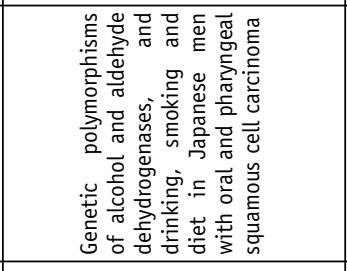 & 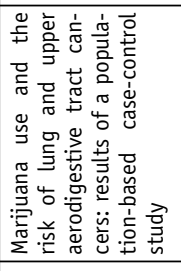 & 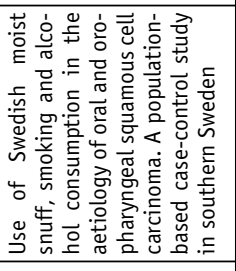 & 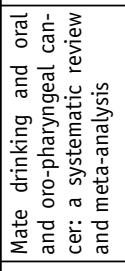 \\
\hline$\sigma$ & 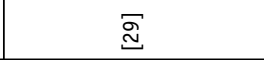 & $\sqrt[\Xi]{ }$ & $\vec{m}$ & 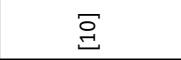 & $\sqrt[\widetilde{\Xi}]{\underline{\Sigma}}$ & চ্口্ \\
\hline
\end{tabular}


Ainsi, la méta-analyse de Hashibe et al. [39] montrait un risque de CCB de 4,8 [intervalle de confiance (IC) à $95 \%: 2,59-8,81$ ] en cas d'exposition au tabac et à l'alcool avec une proportion de cas attribuables à ces deux facteurs de risque seuls ou combinés de 63,7\% [IC à $95 \%: 44,7-74,7]$. Les analyses par niveau de consommation montraient que la proportion de cas attribuables était plus importante chez les personnes consommant quotidiennement plus de trois verres de boisson alcoolisée et fumant plus d'un paquet de cigarettes par jour $(23,5 \%$ [IC à $95 \%: 21,6-24,4])$. En France, l'exposition à ces deux facteurs de risque a varié ces dernières décennies. La prévalence des fumeurs réguliers dans la population générale a diminué chez les hommes de $72 \%$ en 1953 [40] à $31,8 \%$ en 2010 [41] et a augmenté chez les femmes de $17 \%$ en 1953 [40] à 25,7 \% en 2010 [41]. Quant à l'alcool, la consommation moyenne était de 13 litres d'alcool pur par personne en 2006 contre 26 litres en 1961 [42]. En 2005, 21,4\% des hommes et $7,6 \%$ des femmes consommaient quotidiennement de l'alcool [43]. L'exposition à ces deux facteurs de risque est fortement influencée par le niveau socio-économique: le tabagisme régulier et la consommation quotidienne d'alcool sont plus fréquents en France chez les ouvriers et les artisans que chez les cadres, les professions intermédiaires ou les employés [43].

La consommation de fruits et de légumes est associée à un risque moins élevé de cancer, ce que vient confirmer une récente revue s'intéressant spécifiquement à ce facteur [44]. Cette revue citait deux études de cohorte rapportant des risques relatifs de 0,6 (IC à $95 \%: 0,37-0,99$ ) [45] et de 0,7 (IC à $95 \%: 0,55-0,92$ ) [46], pour une consommation de fruits et de légumes importante $(7,7$ et 5,8 portions par jour respectivement) par rapport à une consommation faible $(1,5$ et 2,5 portions par jour). La consommation de café n'était pas associée à la survenue de cancer en général et elle a même plutôt été décrite comme pouvant prévenir certains cancers (endomètre, sein, colon, rectum) [47]. C'est ce qui est rapporté également dans la méta-analyse et la cohorte prospective de la littérature identifiées par notre revue pour les CCB bien qu'il soit difficile de savoir ce qui est anti-carcinogène dans cette boisson ; le modèle animal suggère que la caféine favoriserait plutôt la survenue de cancer [47].

Les HPV sont fortement associés aux cancers de l'oropharynx, cette association est moins évidente pour les autres cancers des voies aérodigestives supérieures [48]. Néanmoins, le rôle oncogène du HPV 16 dans les CCB est reconnu par le Centre International de Recherche sur le Cancer depuis 2007 [49]. Les HPV étaient retrouvés dans 23,5\% des CCB dans une méta-analyse de Kreimer et al. [50]. Plus récemment, une étude française a montré que la prévalence des HPV était de 10,5\% dans les CCB [51]. La vaccination contre les HPV (les types 16 et 18 plus ou moins les types 6 et 11 selon le vaccin) est recommandée en France chez les jeunes filles pour lutter contre le cancer du col de l'utérus [52].
L'efficacité de ce type de vaccin sur la survenue de cancers des VADS n'est actuellement pas connue.

L'étude sur le traumatisme chronique de la muqueuse buccale ne montrait qu'une association entre cancer et présence d'un traumatisme de la tumeur [34]. Il était donc impossible de conclure que le traumatisme était un facteur de risque car les CCB sont le plus souvent exophytiques et découverts lorsqu'ils ont une taille supérieure à $4 \mathrm{~cm}$ (T3). 0 n comprend aisément que cette modification de la muqueuse soit associée à un traumatisme qui n'est probablement que la conséquence du volume de la tumeur. Ceci peut expliquer l'absence de lien dans l'étude entre lésions potentiellement malignes et traumatisme chronique car les lésions potentiellement malignes ne sont pas ou peu exophytiques. Des précisions sur la taille des tumeurs au moment du diagnostic auraient permis une analyse plus fine.

L'association entre mauvaise hygiène bucco-dentaire et CCB observée au moment du diagnostic pourrait aussi être due à un défaut d'hygiène secondaire aux douleurs générées par le cancer. D'autres études sont nécessaires pour confirmer ces résultats. Cependant, l'absence de dents et l'absence de visites régulières chez le chirurgien-dentiste, étaient aussi associées aux CCB, indépendamment de la consommation de tabac [18].

Enfin, l'exposition aux UV était également un facteur de risque retrouvé pour le cancer des lèvres, particulièrement chez les personnes ayant subi une greffe qui sont des personnes à risque pour ce type de cancer [53]. Une étude s'intéressant à de nombreuses expositions professionnelles a montré qu'une exposition aux pesticides était associée à la survenue de CCB, mais seulement si elle était très importante ; cette étude ne précisait pas les types de pesticides incriminés [17]. L'exposition aux pesticides a été mise en cause dans d'autres cancers, comme le cancer de la prostate et le cancer du sein où ces pesticides interviendraient notamment en perturbant les mécanismes endocriniens; il y a peu d'études sur le sujet et les résultats sont contradictoires [54].

La principale limite de cette revue systématique réside dans le choix des mots clés qui, pour des raisons de faisabilité ont été restreints au terme MeSH « mouth neoplasms » mais nous pensons qu'il a permis une recherche relativement exhaustive puisque ce mot clé incluait d'autres termes MeSH comme « oral neoplasms », "mouth cancers », ou " oral cancers ». Néanmoins, une recherche avec des mots clés moins spécifiques comme « head and neck neoplasms » ou des mots libres aurait probablement permis d'obtenir d'autres références, mais leur quantité aurait rendu leur analyse moins précise.

En conclusion, cette revue systématique de la littérature récente sur les facteurs de risque évitables pour les $C C B$, a mis en évidence l'effet synergique de la consommation de tabac et d'alcool favorisant la survenue des CCB ainsi que le rôle associé de facteurs génétiques, comme le polymorphisme du gène codant pour l'alcool déshydrogénase. Une association entre infection à HPV et CCB a été retrouvée, notamment avec les 
types dits à haut risque impliqués dans le cancer du col de l'utérus. Des études ont identifié des facteurs locaux (traumatisme, mauvaise hygiène ...) mais elles ne sont pas apparues suffisamment fiables méthodologiquement pour pouvoir s'appuyer sur leurs conclusions. Les facteurs protecteurs principalement mis en évidence par cette revue étaient une alimentation riche en fruits et légumes frais, la consommation de café et des visites régulières chez le chirurgien-dentiste.

Les CCB constituent un problème de santé publique caractérisé par des changements épidémiologiques majeurs, et une survie qui ne s'améliore pas. Ils sont l'objet de réflexions et de travaux par les institutions et les professionnels de santé car ils sont facilement accessibles à une prévention primaire et secondaire. La prévention primaire, axée aujourd'hui en France sur la lutte contre le tabagisme et la consommation d'alcool, s'intègre dans une démarche de santé publique plus globale puisque ces deux facteurs de risque sont communs à d'autres cancers et à d'autres maladies chroniques non transmissibles fréquentes (maladies cardio-vasculaires, maladies pulmonaires chroniques...). Dans cette logique, le programme de vaccination contre les principaux HPV impliqués dans le cancer du col de l'utérus [52] et le Programme national Nutrition Santé mis en place pour lutter contre l'obésité et les pathologies nutritionnelles [55] auront peut-être un effet favorable sur l'incidence des CCB. En ce qui concerne la prévention secondaire, le dépistage des lésions précancéreuses et des lésions invasives à un stade précoce fait l'objet de mesures dans le Plan Cancer 2009-2013. L'INCa (Institut national du Cancer) a entrepris une politique de sensibilisation des professionnels de santé notamment par la mise en ligne d'un module de formation multimédia [56] et finance, à travers des programmes de recherche interventionnelle, des actions auprès de populations cibles.

\section{Conflits d'intérêt : aucun}

Remerciements. Nous remercions Evelyne Mouillet, responsable du centre de documentation de l'Institut de Santé publique, d'Epidémiologie et de Développement (ISPED) ainsi que les membres de son équipe, Coralie Thore et Christian Weller, pour leur aide lors de la mise en place de la stratégie de recherche et l'obtention des articles.

\section{Références}

1. Petersen PE. Oral cancer prevention and control: the approach of the World Health Organization. Oral Oncol 2009;45:454-60.

2. Ferlay J, Shin HR, Bray F, Forman D, Mathers C, Parkin DM. Estimates of worldwide burden of cancer in 2008: GLOBOCAN 2008. Int J Cancer 2010;127:2893-917.

3. Petersen PE, Bourgeois D, Ogawa H, Estupinan-Day S, Ndiaye C. The global burden of oral diseases and risks to oral health. Bull World Health Organ 2005;83:661-9.
4. Ligier K, Belot A, Launoy G, Velten M, Delafosse P, Guizard AV. Epidemiologie des cancers de la cavité buccale en France. Rev Stomatol Chir Maxillofac 2011;112:164-71.

5. Francim. Survie des patients atteints de cancer en France. Étude des registres de cancers du réseau Francim. Springer-Verlag, Paris, 2007.

6. Salmi L, Pouger P, Spitzer W. Lecture critique et communication médicale scientifique : comment lire, présenter, rédiger et publier une étude clinique ou épidémiologique. Elsevier, Paris, 2002.

7. Richie JP Jr, Kleinman W, Marina P, Abraham P, Wynder EL, Muscat JE. Blood iron, glutathione, and micronutrient levels and the risk of oral cancer. Nutr Cancer 2008;60:474-82.

8. Pintos J, Black MJ, Sadeghi N, Ghadirian P, Zeitouni AG, Viscidi RP, Herrero R, Coutlee F, Franco EL. Human papillomavirus infection and oral cancer: a case-control study in Montreal, Canada. Oral Oncol 2008;44:242-50.

9. Applebaum KM, Furniss CS, Zeka A, Posner MR, Smith JF, Bryan $\mathrm{J}$, Eisen EA, Peters ES, McClean MD, Kelsey KT. Lack of association of alcohol and tobacco with HPV16-associated head and neck cancer. J Natl Cancer Inst 2007;99:1801-10.

10. Hashibe M, Morgenstern H, Cui Y, Tashkin DP, Zhang ZF, Cozen W, Mack TM, Greenland S. Marijuana use and the risk of lung and upper aerodigestive tract cancers: results of a population-based case-control study. Cancer Epidemiol Biomark Prev 2006; 15:1829-34.

11. Supic G, Jovic N, Kozomara R, Zeljic K, Magic Z. Interaction between the MTHFR C677T polymorphism and alcohol--impact on oral cancer risk and multiple DNA methylation of tumor-related genes. J Dent Res 2011;90:65-70.

12. Kenborg $L$, Jorgensen AD, Budtz-Jorgensen $E$, Knudsen $L E$, Hansen J. Occupational exposure to the sun and risk of skin and lip cancer among male wage earners in Denmark: a populationbased case-control study. Cancer Causes Control 2010;21:134755.

13. Sapkota A, Hsu CC, Zaridze D, Shangina 0, Szeszenia-Dabrowska N, Mates D, Fabianova E, Rudnai P, Janout V, Holcatova I, Brennan $P$, Boffetta P, Hashibe M. Dietary risk factors for squamous cell carcinoma of the upper aerodigestive tract in central and eastern Europe. Cancer Causes Control 2008;19:1161-70.

14. Garavello W, Giordano L, Bosetti C, Talamini R, Negri E, Tavani A, Maisonneuve $P$, Franceschi S, La Vecchia C. Diet diversity and the risk of oral and pharyngeal cancer. Eur J Nutr 2008;47:280-4.

15. Galeone C, Pelucchi C, Talamini R, Levi F, Bosetti C, Negri E, Franceschi S, La Vecchia C. Role of fried foods and oral/pharyngeal and oesophageal cancers. Br J Cancer 2005;92:2065-9.

16. Rossi M, Garavello W, Talamini R, Negri E, Bosetti C, Dal Maso L, Lagiou P, Tavani A, Polesel J, Barzan L, Ramazzotti V, Franceschi $S$, La Vecchia C. Flavonoids and the risk of oral and pharyngeal cancer: a case-control study from Italy. Cancer Epidemiol Biomark Prev 2007;16:1621-5.

17. Tarvainen L, Kyyronen P, Kauppinen T, Pukkala E. Cancer of the mouth and pharynx, occupation and exposure to chemical agents in Finland [in 1971-95]. Int J Cancer 2008;123:653-9.

18. Rosenquist K, Wennerberg J, Schildt EB, Bladstrom A, Goran Hansson B, Andersson G. Oral status, oral infections and some lifestyle factors as risk factors for oral and oropharyngeal 
squamous cell carcinoma. A population-based case-control study in southern Sweden. Acta Otolaryngol 2005;125:1327-36.

19. Canova C, Richiardi L, Merletti F, Pentenero M, Gervasio C, Tanturri G, Garzino-Demo P, Pecorari G, Talamini R, Barzan L, Sulfaro S, Franchini G, Muzzolini C, Bordin S, Pugliese GN, Macri E, Simonato L. Alcohol, tobacco and genetic susceptibility in relation to cancers of the upper aerodigestive tract in northern Italy. Tumori 2010;96:1-10.

20. Weikert $C$, Dietrich $T$, Boeing $H$, Bergmann MM, Boutron-Ruault MC, Clavel-Chapelon F, Allen N, Key T, Lund E, Olsen A, Tjonneland A, Overvad K, Rohrmann S, Linseisen J, Pischon T, Trichopoulou A, Weinehall L, Johansson I, Sanchez MJ, Agudo A, Barricarte A, Amiano $P$, Chirlaque MD, Quiros JR, Wirfalt E, Peeters PH, Buenode-Mesquita HB, Vrieling A, Pala V, Palli D, Vineis P, Tumino R, Panico S, Bingham S, Khaw KT, Norat T, Jenab M, Ferrari P, Slimani $\mathrm{N}$, Riboli E. Lifetime and baseline alcohol intake and risk of cancer of the upper aero-digestive tract in the European Prospective Investigation into Cancer and Nutrition (EPIC) study. Int J Cancer 2009;125:406-12.

21. Bosetti C, Gallus S, Peto R, Negri E, Talamini R, Tavani A, Franceschi S, La Vecchia C. Tobacco smoking, smoking cessation, and cumulative risk of upper aerodigestive tract cancers. Am J Epidemiol 2008;167:468-73.

22. Polesel J, Talamini R, La Vecchia C, Levi F, Barzan L, Serraino D, Franceschi S, Dal Maso L. Tobacco smoking and the risk of upper aero-digestive tract cancers: A reanalysis of case-control studies using spline models. Int J Cancer 2008;122:2398-402.

23. Rosenquist K, Wennerberg J, Schildt EB, Bladstrom A, Hansson $B G$, Andersson G. Use of Swedish moist snuff, smoking and alcohol consumption in the aetiology of oral and oropharyngeal squamous cell carcinoma. A population-based case-control study in southern Sweden. Acta Otolaryngol 2005;125:991-8.

24. Naganuma T, Kuriyama S, Kakizaki M, Sone T, Nakaya N, OhmoriMatsuda K, Nishino Y, Fukao A, Tsuji I. Coffee consumption and the risk of oral, pharyngeal, and esophageal cancers in Japan: the Miyagi Cohort Study. Am J Epidemiol 2008;168:1425-32.

25. Ide R, Fujino $Y$, Hoshiyama $Y$, Mizoue T, Kubo T, Pham TM, Shirane K, Tokui N, Sakata K, Tamakoshi A, Yoshimura T. A prospective study of green tea consumption and oral cancer incidence in Japan. Ann Epidemiol 2007;17:821-6.

26. Cancela M de C, Ramadas K, Fayette JM, Thomas G, Muwonge R, Chapuis F, Thara S, Sankaranarayanan R, Sauvaget C. Alcohol intake and oral cavity cancer risk among men in a prospective study in Kerala, India. Community Dent Oral Epidemiol 2009;37:342-9.

27. Subapriya R, Thangavelu A, Mathavan B, Ramachandran CR, Nagini S. Assessment of risk factors for oral squamous cell carcinoma in Chidambaram, Southern India: a case-control study. Eur J Cancer Prev 2007;16: 251-6.

28. Jayalekshmi PA, Gangadharan P, Akiba S, Nair RR, Tsuji M, Rajan B. Tobacco chewing and female oral cavity cancer risk in Karunagappally cohort, India. Br J Cancer 2009;100:848-52.

29. Muwonge R, Ramadas K, Sankila R, Thara S, Thomas G, Vinoda J, Sankaranarayanan R. Role of tobacco smoking, chewing and alcohol drinking in the risk of oral cancer in Trivandrum, India: a nested case-control design using incident cancer cases. Oral Oncol 2008;44:446-54.
30. Rahman M, Sakamoto J, Fukui T, Calculation of population attributable risk for bidi smoking and oral cancer in south Asia. Prev Med 2005;40: 510-4.

31. Asakage T, Yokoyama A, Haneda T, Yamazaki M, Muto M, Yokoyama T, Kato H, Igaki $H$, Tsujinaka T, Kumagai $Y$, Yokoyama M, Omori T, Watanabe H. Genetic polymorphisms of alcohol and aldehyde dehydrogenases, and drinking, smoking and diet in Japanese men with oral and pharyngeal squamous cell carcinoma. Carcinogenesis 2007;28:865-74.

32. Ide R, Mizoue T, Fujino Y, Hoshiyama Y, Sakata K, Tamakoshi A, Yoshimura T. Cigarette smoking, alcohol drinking, and oral and pharyngeal cancer mortality in Japan. Oral Dis 2008;14:314-9.

33. van Leeuwen MT, Grulich AE, McDonald SP, McCredie MR, Amin J, Stewart JH, Webster AC, Chapman JR, Vajdic CM. Immunosuppression and other risk factors for lip cancer after kidney transplantation. Cancer Epidemiol Biomark Prev 2009;18:561-9.

34. Piemonte ED, Lazos JP, Brunotto M. Relationship between chronic trauma of the oral mucosa, oral potentially malignant disorders and oral cancer. J Oral Pathol Med 2010;39:513-7.

35. De Stefani E, Boffetta P, Ronco AL, Correa P, Oreggia F, DeneoPellegrini $\mathrm{H}$, Mendilaharsu M, Leiva J. Dietary patterns and risk of cancer of the oral cavity and pharynx in Uruguay. Nutr Cancer 2005;51:132-9.

36. Dasanayake AP, Silverman AJ, Warnakulasuriya S. Mate drinking and oral and oro-pharyngeal cancer: a systematic review and meta-analysis. Oral Oncol 2010;46:82-6.

37. Galeone C, Tavani A, Pelucchi C, Turati F, Winn DM, Levi F, Yu GP, Morgenstern H, Kelsey K, Dal Maso L, Purdue MP, McClean M, Talamini R, Hayes RB, Franceschi S, Schantz S, Zhang ZF, Ferro G, Chuang SC, Boffetta P, La Vecchia C, Hashibe M. Coffee and tea intake and risk of head and neck cancer: pooled analysis in the international head and neck cancer epidemiology consortium. Cancer Epidemiol Biomark Prev 2010;19:1723-36.

38. Lubin JH, Gaudet MM, Olshan AF, Kelsey K, Boffetta P, Brennan P, Castellsague X, Chen C, Curado MP, Dal Maso L, Daudt AW, Fabianova E, Fernandez L, Wunsch-Filho V, Franceschi S, Herrero R, Koifman S, La Vecchia C, Lazarus P, Levi F, Lissowska J, Mates IN, Matos E, McClean M, Menezes A, Morgenstern H, Muscat J, Eluf Neto J, Purdue MP, Rudnai P, Schwartz SM, Shangina 0, Sturgis EM, Szeszenia-Dabrowska N, Talamini R, Wei Q, Winn D, Zhang ZF, Hashibe M, Hayes RB. Body mass index, cigarette smoking, and alcohol consumption and cancers of the oral cavity, pharynx, and larynx: modeling odds ratios in pooled case-control data. Am J Epidemiol 2010;171:1250-61.

39. Hashibe M, Brennan P, Chuang SC, Boccia S, Castellsague $X$, Chen C, Curado MP, Dal Maso L, Daudt AW, Fabianova E, Fernandez L, Wunsch-Filho V, Franceschi S, Hayes RB, Herrero R, Kelsey K, Koifman S, La Vecchia C, Lazarus P, Levi F, Lence JJ, Mates D, Matos E, Menezes A, McClean MD, Muscat J, Eluf-Neto J, Olshan $A F$, Purdue M, Rudnai P, Schwartz SM, Smith E, Sturgis EM, Szeszenia-Dabrowska N, Talamini R, Wei Q, Winn DM, Shangina 0, Pilarska A, Zhang ZF, Ferro G, Berthiller J, Boffetta P. Interaction between tobacco and alcohol use and the risk of head and neck cancer: pooled analysis in the International Head and Neck Cancer Epidemiology Consortium. Cancer Epidemiol Biomark Prev 2009;18:541-50. 
40. Hill C. Trends in tobacco smoking and consequences on health in France. Prev Med 1998;27:514-9.

41. Beck F, Guignard R, Richard J, Wilquin J, Peretti-Wattel R.

42. Observatoire français des drogues et des toxicomanies. Séries statistiques alcool. 2010 ; Acces le 17/11/2011. Disponible sur : http://www.ofdt.fr/BDD_len/seristat/00014.xhtml.

43. Beck F, Guilbert $P$, Gautier A. Baromètre santé 2005 - Attitudes et comportements de santé : INPES, coll. Baromètres santé, 2007.

44. Key TJ. Fruit and vegetables and cancer risk. $\mathrm{Br} \mathrm{J}$ Cancer 2011;104:6-11.

45. Boeing $H$, Dietrich $T$, Hoffmann $K$, Pischon $T$, Ferrari $P$, Lahmann $\mathrm{PH}$, Boutron-Ruault MC, Clavel-Chapelon F, Allen N, Key T, Skeie $G$, Lund $E$, Olsen A, Tjonneland A, Overvad K, Jensen MK, Rohrmann S, Linseisen J, Trichopoulou A, Bamia C, Psaltopoulou $T$, Weinehall $L$, Johansson Sanchez MJ, Jakszyn P, Ardanaz E, Amiano P, Chirlaque MD, Quiros JR, Wirfalt E, Berglund G, Peeters $\mathrm{PH}$, van Gils $\mathrm{CH}$, Bueno-de-Mesquita $\mathrm{HB}$, Buchner $\mathrm{FL}$, Berrino $\mathrm{F}$, Palli D, Sacerdote C, Tumino R, Panico S, Bingham S, Khaw KT, Slimani N, Norat T, Jenab M, Riboli E. Intake of fruits and vegetables and risk of cancer of the upper aero-digestive tract: the prospective EPIC-study. Cancer Causes Control 2006;17:957-69.

46. Freedman ND, Park Y, Subar AF, Hollenbeck AR, Leitzmann MF, Schatzkin A, Abnet CC. Fruit and vegetable intake and head and neck cancer risk in a large United States prospective cohort study. Int J Cancer 2008;122:2330-6.

47. Arab L. Epidemiologic evidence on coffee and cancer. Nutr Cancer 2010;62:271-83.

48. D'Souza G, Dempsey A. The role of HPV in head and neck cancer and review of the HPV vaccine. Prev Med 2011;53(Suppl 1):S5S11.
49. IARC monograph working group. Human papillomaviruses (p. 670). In IARC onographs on the evaluation of carcinogenic risks to humans. IARC Press, Lyon, 2007.

50. Kreimer R, Clifford GM, Boyle P, Franceschi S. Human papillomavirus types in head and neck squamous cell carcinomas worldwide: a systematic review. Cancer Epidemiol Biomarkers Prev 2005;14:467-75.

51. St Guily JL, Jacquard AC, Pretet JL, Haesebaert J, Beby-Defaux A, Clavel C, Agius G, Birembaut P, Okais C, Leocmach Y, Soubeyrand B, Pradat P, Riethmuller D, Mougin C, Denis F. Human papillomavirus genotype distribution in oropharynx and oral cavity cancer in France--The EDiTH VI study. J Clin Virol 2011;51:100-4.

52. Haute Autorité de Santé. Recommandations du collège de la HAS sur les vaccins préventifs de l'infection par les papillomavirus (HPV) - Gardasil et Cervarix 2008; Acces le 17/11/2011. Disponible sur : http://www.has-sante.fr/portail/jcms/c_656898/ recommandation-du-college-de-la-has-sur-les-vaccinsanti-hpv.

53. Makitie AA, Lundberg M, Salmela K, Kyllonen L, Pukkala E. Head and neck cancer in renal transplant patients in Finland. Acta Otolaryngol 2008;128:1255-8.

54. Weichenthal S, Moase C, Chan P. A review of pesticide exposure and cancer incidence in the Agricultural Health Study cohort. Environ Health Perspect 2010;118:1117-25.

55. Programme national Nutrition Santé. Les objectifs de santé publique. ND ; Acces le 17/11/2011. Disponible sur : http:// www.mangerbouger.fr/pnns/les-objectifs-de-sante-publique.html.

56. Agence nationale sanitaire et scientifique en cancérologie. Un module de formation multimédia. 2009 ; Acces le 17/11/2011. Disponible sur: http://www.e-cancer.fr/depistage/cancerscavite-buccale/module-de-formation-multimedia. 